\title{
Synthesis and antibacterial activity of novel 11-[3-[(arylcarbamoyl)oxy]propylamino]-11-deoxy-6- O-methyl-3-oxoerythromycin A 11-N,12-O-cyclic carbamate derivatives
}

\author{
Zhonghui Zheng ${ }^{1}$, Deping Du ${ }^{1}$, Lili Cao ${ }^{1}$, Jun $\mathrm{Liu}^{2}$ and Xiaofang Chen ${ }^{2}$ \\ A series of novel 11-[3-[(arylcarbamoyl)oxy]propylamino]-11-deoxy-6-O-methyl-3-oxoerythromycin A 11-N,12-O-cyclic carbamate \\ derivatives (6a-h) were designed, synthesized and evaluated for their antibacterial activities in vitro. Most of these compounds \\ had significant antibacterial activity against two groups of pathogens of Methicillin-sensitive Staphylococcus aureus \\ $\left(\mathrm{MIC}_{50}=0.031-2 \mu \mathrm{g} \mathrm{ml}^{-1}\right.$ ) except $6 \mathrm{~g}$ and Methicillin-sensitive S. epidermidis $\left(\mathrm{MIC}_{50}=0.031-0.5 \mu \mathrm{g} \mathrm{ml}^{-1}\right)$. MIC 90 of $6 \mathrm{~d}$ \\ against Methicillin-resistant $S$. epidermidis was at least 16 -fold better than that of erythromycin (EMA), azithromycin (AZM) and \\ ABT-773. $6 \mathrm{~d}$ and $6 \mathrm{e}$ had more potent antibacterial activity against $S$. pneumoniae than EMA, AZM and ABT-773. In particular, \\ compounds $6 \mathrm{~d}$ and $6 \mathrm{e}$ also showed relatively potent activity against Haemophilus influenzae and Streptococcus hemolyticus. \\ The Journal of Antibiotics (2016) 69, 811-817; doi:10.1038/ja.2016.42; published online 27 April 2016
}

\section{INTRODUCTION}

The rapid development of antibiotics-resistant strains such as Methicillin-resistant Staphylococcus aureus, Vancomycin-resistant Enterococcus, Penicillin-resistant S. pneumoniae, has created a serious problem for the clinical treatment in recent years. ${ }^{1}$ In a research on clinic ophthalmic bacteria, $87 \%$ of the Methicillin-resistant Staphylococcus aureus isolates were resistant to azithromycin (AZM) and $42 \%$ of the Methicillin-resistant S. epidermidis (MRSE) strains tested were resistant to AZM, respectively. ${ }^{2}$ The clinically relevant coagulase-negative Staphylococcal species, S. epidermidis are among the leading causes of nosocomial infections in humans, particularly in neonates, immunocompromised patients and patients with indwelling and implanted devices. ${ }^{3}$ Farrell et al. ${ }^{4}$ reported that resistant S. pneumoniae possessing both erm and mef genes markedly increase in only 3 years in the United States. Therefore, there is an urgent need for novel macrolide antibiotics to suppress the resistance. Macrolides antibiotics, which are active against Gram-positive bacteria, especially S. pneumoniae, belong to one of the most commonly used families of clinically important antibiotics.

Erythromycin A (EMA), the representative of first-generation macrolides, has been widely used to treat respiratory infections for almost 60 years. However, EMA is prone to degrade under acidic conditions, leading to the undesirable gastrointestinal side effects and low bioavailability. With the purpose of improving the acid stability, second-generation macrolides, including clarithromycin and AZM
(Figure 1), have been developed and widely prescribed for upper and lower respiratory tract infections., ${ }^{5,6}$ These compounds demonstrated a broader spectrum of antibacterial activity, better pharmacokinetic properties and fewer gastrointestinal side effects as compared with EMA. Unfortunately, the therapeutic utility of these macrolides has resulted in the increasing emergence of resistant pathogens. The two primary mechanisms of macrolide resistance are mediated by erm-encoded methylation of $23 \mathrm{~S}$ ribosomal RNA and mef-encoded efflux, respectively. ${ }^{7,8}$

To overcome the growing problems of macrolide resistance, the research has focused on the discovery of novel macrolides with greater efficacy and safety. The third-generation macrolides, ketolides such as telithromycin and cethromycin (Figure 1), ${ }^{9,10}$ displayed improved activity against some of the resistant strains and may offer alternative therapy for Gram-positive infections attributable to resistant pathogens. Both telithromycin and cethromycin share the same key structure features, including 3-keto group, 11,12-cyclic carbamate and a proper hetero-arylalkyl side chain, which is essential for interacting with the $23 \mathrm{~S}$ ribosomal RNA of bacteria. ${ }^{11,12}$

Recently, intensive research focusing on the structural modifications of the ketolide core skeleton in search of novel potent ketolides has yielded many ketolides derivatives, such as 6-O-alkyl ketolides, ${ }^{10,13-15}$ 9-oxime macrolides, ${ }^{16-18}$ 11,12-cyclic carbamate ketolides, ${ }^{19-21}$ 13-substituents ketolides and 6,11-bridged ketolides. ${ }^{22-24}$ Herein, we described the synthesis and in vitro biological evaluation of a set of

\footnotetext{
${ }^{1}$ Shandong Xinhua Pharmaceutical Co., Ltd, Zibo City, Shandong Province, China and ${ }^{2}$ Institute of Medicinal Biotechnology, Chinese Academy of Medical Sciences \& Peking Union Medical College, Beijing, China

Correspondence: Dr Z Zheng or Dr X Chen, Institute of Medicinal Biotechnology, Chinese Academy of Medical Sciences \& Peking Union Medical College, tiantanxli 1\#, Beijing 100050, China.

E-mail: zhengzhonghui@sina.com or chenchemistry@hotmail.com

Received 12 October 2015; revised 24 February 2016; accepted 14 March 2016; published online 27 April 2016
} 


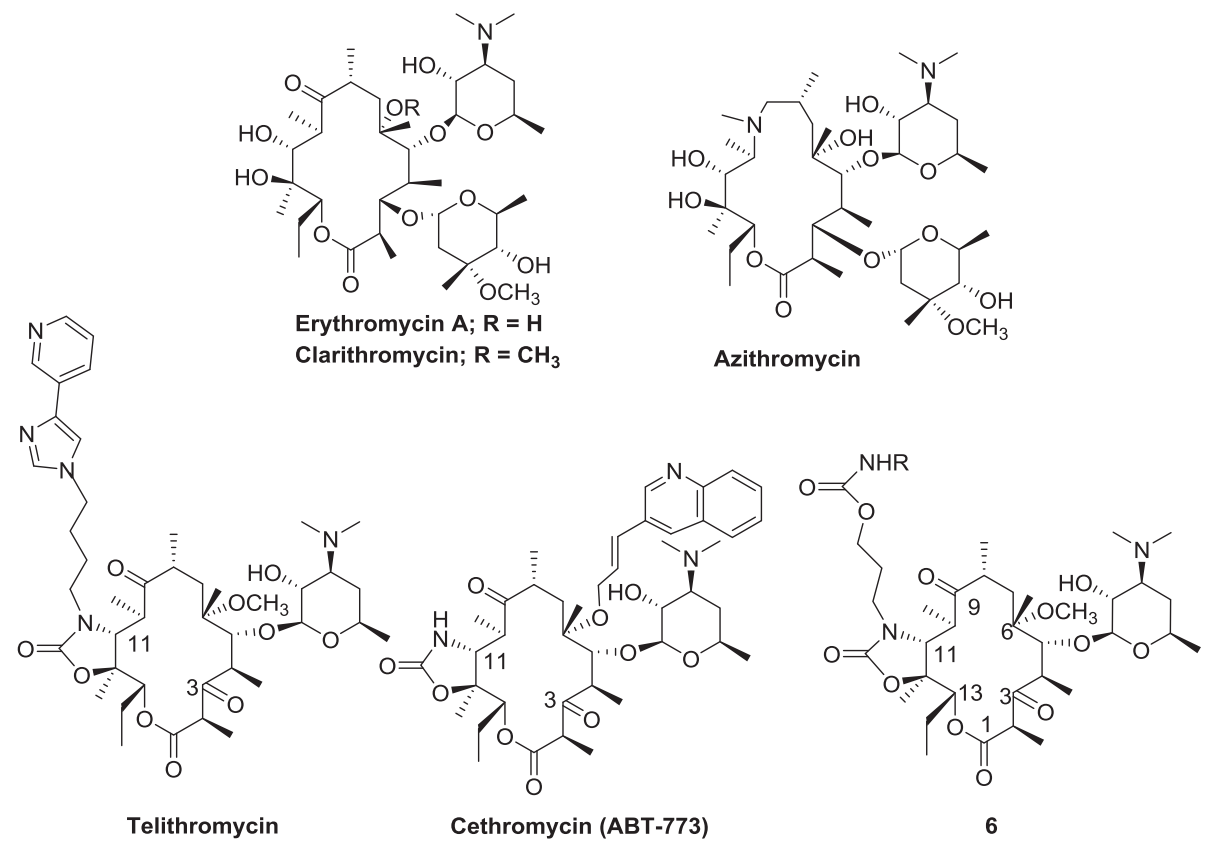

Figure 1 Structures of erythromycin, clarithromycin, azithromycin, telithromycin and cethromycin (ABT-773).

novel 14-membered macrolide analogs that comprised the essential features for addressing macrolide resistance especially by erm-encoded methylation of $23 \mathrm{~S}$ ribosomal RNA. By introducing various substitutedphenyl carbamate propyl chain to the $11-\mathrm{N}$ of the 11-amino-11-deoxy-3des(hexopyranosyloxy)-6-O-methyl-3-oxoerythromycin A 11- $\mathrm{N}, 12-\mathrm{O}$ cyclic carbamate, a series of novel 11-N,12-O-cyclic carbamate ketolides were obtained.

\section{RESULTS AND DISCUSSION}

A simple and efficient method for preparation of novel 14-membered macrolide analogs was developed. The objective compounds were evaluated for antibacterial activity against macolide-susceptible and -resistant pathogens. Most of the compounds tested showed potent activity against most resistant bacteria, indicating that the introduction of phenyl substituents to the $11-N$ position can markedly enhance the antibacterial activity. Accounding to the structure-activity relationship studies it is demonstrated that 11,12-cyclic carbamate would increase the stability of the conformation of the ketolides, and meanwhile might be beneficial for the activitiy against macrolide-susceptible and -resistant bacteria. Accordingly, we hoped that introducing a carbamate group to the $11-N$-alkyl position might remarkbly increase the antibacterial activity. However, almost all of the compounds did not possess better antibacterial activities than those of compounds with alkyl substituents at the $11-N$ position.

In general, the derivative $\mathbf{6 a}$ without substituents on the phenyl ring was less active than those containing substituents except $\mathbf{6 g}$. The position and properties of substituents on the benzene ring had great influence on the in vitro antibacterial activity. In the 4 -substitution, a comparison among $\mathbf{6 b}, \mathbf{6 c}, \mathbf{6 d}$ and $\mathbf{6 h}$ indicated that electrical property had little effect on the activity. $\mathrm{MIC}_{90}$ of $\mathbf{6 d}$ with 4-nitro substituent against MRSE was better than other derivatives with the 4-substitution group. $\mathbf{6 h}$ had the least activity against $S$. pneumoniae among the four compounds. In contrast, the derivatives 6e and $6 \mathrm{~g}$ with substitution at the 3-position of the phenyl ring displayed a great difference on antibacterial activity. 6e with a methyl group was much powerful than $\mathbf{6 g}$ containing a chloro group. This

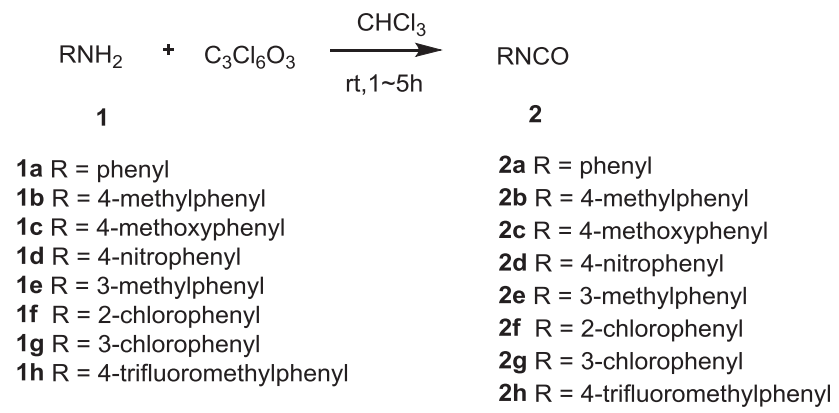

Scheme 1 Synthesis of a set of isocyanate $(2 \mathbf{a}-\mathbf{h})$.

result suggested that the 3 -substituent was very important for the activity. In addition, $6 \mathbf{f}$ with a chloro group substitution in the 2position showed more potent activity than $\mathbf{6 g}$. These results indicated the importance of the presence and location of the functional groups for activity. Further research on the structure-activity relationship is necessary especially on 3-postion substitution, which maybe is useful for designing new ketolides with broader antibacterial spectrum. On the whole, 6d and $\mathbf{6 e}$ showed potent activity against MSSA-EAR, MRSE and Haemophilus influenzae, whereas $\mathbf{6 g}$ was the least active compound.

\section{Chemistry}

The synthetic method of aryl isocyanates (2) is shown in Scheme 1. Condensation of $\mathbf{1}$ with bis(trichloromethyl) carbonate gave the desired aryl isocyanates $(\mathbf{2} \mathbf{a}-\mathbf{h})$. Scheme 2 describes the synthesis of 11-[3-[(arylcarbamoyl)oxy] propylamino]-11-deoxy-3-des(hexopyranosyloxy)-6-O-methyl-3-oxoerythromycin A 11-N,12-O-cyclic carbamate (6) starting from 2'-O-benzoyl-10,11-dehydro-3-des(hexopyranosyloxy)-12-O-imidazolylcarbonyl-6-O-methyl-3-oxoerythromycin A (3). Treatment of 3 with 1-amino propanol in N,N-Dimethylformamide (DMF) at room temperature afforded compound 4 . Compounds (6a-h) were prepared by reacting compound 4 with corresponding aryl 

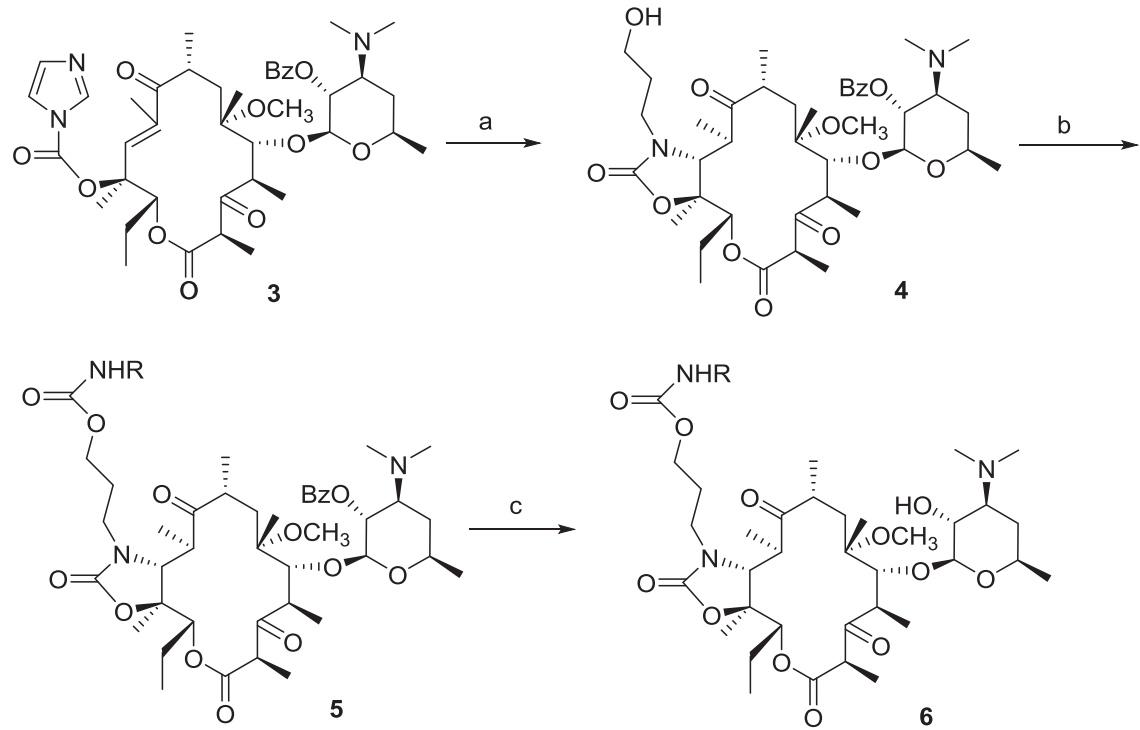

Scheme 2 Reagents and conditions: (a) $\mathrm{NH}_{2} \mathrm{CH}_{2} \mathrm{CH}_{2} \mathrm{CH}_{2} \mathrm{OH}, \mathrm{N}, \mathrm{N}$-Dimethylformamide (DMF), room temperature (rt), $62 \mathrm{~h}$, $85 \%$; (b) dicyclohexylcarbodiimide (DCC), 4-dimethylaminopyridine (DMAP), RNCO is $\mathrm{R}-\mathrm{N}=\mathrm{C}=\mathrm{O}$, DMAP, $\mathrm{CH}_{2} \mathrm{Cl}_{2}$, rt, $20 \mathrm{~h}, 75-90 \%$; (c) $\mathrm{CH}_{3} \mathrm{OH}$, reflux, $20 \mathrm{~h}, 90 \sim 98 \%$.

Table 1 In vitro antibacterial activity (MIC $\left(\mu \mathrm{g} \mathrm{ml}^{-1}\right)$ ) of prepared macrolides against $S$. aureus and $S$. epidermidis

\begin{tabular}{|c|c|c|c|c|c|c|c|c|c|c|}
\hline \multirow[b]{2}{*}{ Compound/strain } & \multicolumn{2}{|c|}{$M S S A-E A S^{a}$} & \multicolumn{2}{|c|}{$M S S A-E A R^{b}$} & \multicolumn{2}{|c|}{$M R S A^{\mathrm{C}}$} & \multicolumn{2}{|c|}{$M R S E^{\mathrm{d}}$} & \multicolumn{2}{|c|}{$M S S E^{\mathrm{e}}$} \\
\hline & $M I C_{50}$ & $M I C_{90}$ & $M I C_{50}$ & $M I C_{90}$ & $M I C_{50}$ & $M I C_{90}$ & $M I C_{50}$ & $M I C_{90}$ & $M I C_{50}$ & $M I C_{90}$ \\
\hline $6 b$ & 0.062 & 0.125 & 0.125 & 0.125 & 128 & 256 & 0.062 & 256 & 0.062 & 0.062 \\
\hline $6 c$ & 0.031 & 0.125 & 0.125 & 0.25 & 256 & $>256$ & 16 & 32 & 0.031 & 0.062 \\
\hline $6 d$ & 0.25 & 0.25 & 0.5 & 0.5 & $>256$ & $>256$ & 1 & 8 & 0.25 & 0.25 \\
\hline $6 g$ & 0.25 & 0.5 & $>256$ & $>256$ & $>256$ & $>256$ & $>256$ & $>256$ & 0.25 & 0.25 \\
\hline $6 \mathrm{~h}$ & 0.062 & 0.25 & 0.062 & 2 & 256 & $>256$ & 2 & 256 & 0.125 & 0.5 \\
\hline 4 & 0.5 & 0.5 & 0.5 & 1 & $>256$ & $>256$ & 2 & $>256$ & 0.25 & 0.25 \\
\hline ABT-773 & $<0.016$ & $<0.016$ & $<0.016$ & $<0.016$ & 256 & $>256$ & $<0.016$ & 128 & $<0.016$ & $<0.016$ \\
\hline EMA & 0.125 & 0.25 & $>256$ & $>256$ & $>256$ & $>256$ & $>256$ & $>256$ & 0.125 & 0.125 \\
\hline
\end{tabular}

Abbreviations: ABT-773, cethromycin; AZM, azithromycin; EMA, erythromycin; MRSA, methicillin resistant Staphylococcus aureus; MRSE, methicillin resistant Staphylococcus epidermidis; MSSA-EAS, methicillin susceptible Staphylococcus aureus and erythromycin susceptible; MSSA-EAR, methicillin susceptible Staphylococcus aureus and erythromycin resistant; MSSE, methicillin susceptible Staphylococcus epidermidis.

a10 strains for MSSA-EAS.

b10 strains for MSSA-EAR.

c10 strains for MRSA.

d10 strains for MRSE.

e 10 strains for MSSE.

isocyanates $(\mathbf{2} \mathbf{a}-\mathbf{h})$ in the presence of dicyclohexylcarbodiimide (DCC) and 4-dimethylaminopyridine (DMAP) at room temperature, ${ }^{25}$ followed by deprotection of the benzoyl group with methanol.

\section{Antibacterial activity}

The 11-[3-[(arylcarbamoyl)oxy]propylamino]-11-deoxy-6-O-methyl3-oxoerythromycin A 11-N,12-O-cyclic carbamate derivatives, as well as cethromycin (ABT-773), EMA, azithromycin as references, were tested for in vitro antibacterial activity against three groups of S. aureus, two groups of S. epidermis, Enterococcus faecalis, Streptococcus pneumoniae, Haemophilus influenzae and Streptococcus hemolyticus. The activities are reported in Tables 1 and 2 MICs determined using the broth microdilution method.
The results in Tables 1 and 2 show the antibacterial activity of 11[3-[(arylcarbamoyl)oxy]propylamino]-11-deoxy-6-O-methyl-3-oxoerythromycin A 11-N,12-O-cyclic carbamate derivatives and reference compounds (ABT-773, EMA and $\mathrm{AZM}$ ). $\mathrm{MIC}_{50}$ (the concentration in which growth of $50 \%$ of strains were inhibited) and $\mathrm{MIC}_{90}$ (the concentration in which $90 \%$ of strains were inhibited) were tested. All of the tested compounds except $\mathbf{6 g}$ proved to have good antibacterial activity against MSSA $\left(\right.$ MIC $\left._{50}=0.031-2 \mu \mathrm{g} \mathrm{ml}^{-1}\right)$, MSSE $\quad\left(\mathrm{MIC}_{50}=0.031-0.5 \mu \mathrm{g} \mathrm{ml}^{-1}\right)$, Streptococcus pneumoniae $\left(\mathrm{MIC}_{50}=0.062-16 \mu \mathrm{g} \mathrm{ml}^{-1}\right)$. The MIC 90 of $\mathbf{6 d}$ for MRSE were 2-16 times better than EMA, AZM and ABT-773. Compounds $\mathbf{6 d}$ and $\mathbf{6 e}$ had more potent antibacterial activity against Streptococcus pneumoniae than the reference compounds EMA, AZM and ABT-773. Specifically, 
Table 2 In vitro antibacterial activity (MIC ( $\left.\mu \mathrm{g} \mathrm{mL}^{-1}\right)$ ) of prepared macrolides against other strains

\begin{tabular}{|c|c|c|c|c|c|c|c|c|}
\hline \multirow{2}{*}{$\begin{array}{l}\text { Compound/ } \\
\text { strain }\end{array}$} & \multicolumn{2}{|c|}{$\begin{array}{c}\text { Enterococcus } \\
\text { faecalis }^{\mathrm{a}}\end{array}$} & \multicolumn{2}{|c|}{$\begin{array}{l}\text { Streptococcus } \\
\text { pneumoniae }^{b}\end{array}$} & \multicolumn{2}{|c|}{$\begin{array}{c}\text { Haemophilus } \\
\text { influenzae }\end{array}$} & \multicolumn{2}{|c|}{$\begin{array}{l}\text { Streptococcus } \\
\text { hemolyticus }^{\mathrm{d}}\end{array}$} \\
\hline & $M I C_{50}$ & $M I C_{90}$ & $M I C_{50}$ & $M I C_{90}$ & $M I C_{50}$ & $M C_{90}$ & $M I C_{50}$ & $M^{\prime} C_{90}$ \\
\hline $6 a$ & $>256$ & $>256$ & 16 & 32 & 32 & 32 & 4 & 32 \\
\hline $6 b$ & $>256$ & $>256$ & 0.5 & 2 & 16 & 64 & 1 & 2 \\
\hline $6 c$ & $>256$ & $>256$ & 0.25 & 2 & 16 & 32 & 2 & 16 \\
\hline $6 d$ & $>256$ & $>256$ & 0.125 & 0.25 & 4 & 8 & 2 & 2 \\
\hline $6 e$ & $>256$ & $>256$ & 0.062 & 0.125 & 4 & 8 & 1 & 2 \\
\hline $6 f$ & $>256$ & $>256$ & 2 & 8 & 64 & 128 & 8 & 8 \\
\hline $6 h$ & $>256$ & $>256$ & 4 & 32 & 32 & 64 & 2 & 4 \\
\hline 4 & $>256$ & $>256$ & 4 & 8 & 16 & 16 & 2 & 8 \\
\hline ABT-773 & 32 & 32 & 0.5 & 1 & 1 & 2 & 1 & 2 \\
\hline EMA & $>256$ & $>256$ & 2 & 4 & 4 & 8 & 2 & 4 \\
\hline AZM & $>256$ & $>256$ & 1 & 2 & 2 & 8 & 16 & 16 \\
\hline
\end{tabular}

${ }^{a} 10$ strains for $E$. faecalis.

b10 strains for S. pneumoniae.

c10 strains for $H$. influenzae.

${ }^{d} 10$ strains for S. hemolyticus.

compounds 6d and 6e showed more potent activity against S. hemolyticus than EMA and AZM, which was similar with ABT-773.

\section{EXPERIMENTAL PROCEDURE}

All necessary solvents were purified before use, unless noted otherwise. Reactions were monitored by TLC using $0.20-\mathrm{mm}$ pre-coated silica gel plates Merck DC-alurolle Kieselgel 60GF254. Column chromatography was performed with the indicated solvents using silica gel $\mathrm{H}$ (Qingdao Marine chemical plant, Shandong, China). IR spectra were recorded on KBr pellets using a Shimadzu IR-435 spectrometer. ${ }^{1} \mathrm{H}$ NMR spectra were recorded on a WYS-300 or VarianX1-400 or Inova-500 spectrometer at ambient temperature (TMS as internal standard of chemical shifts). Mass spectra were obtained on a ZAB-2F or Autospect-Ultima ETOF mass spactrometer for FAB-MS or ESI-MS. Melting points are uncorrected and were determined on an X-6 or RY-1 melting point apparatus. The starting material, 2'-O-benzoyl-10,11-dehydro-3des(hexopyranosyloxy)-12-O-imidazolylcarbonyl-6-O-methyl-3-oxoerythromycin A (3), was prepared according to the published procedure. ${ }^{26}$

\section{General methods for aryl isocyanate (2a-h)}

To a solution of bis(trichloromethyl) carbonate in $\mathrm{CHCl}_{3}$ was added dropwise aromatic amines in $\mathrm{CHCl}_{3}$ in an ice bath. When the addition was finished, the reaction mixture was stirred at room temperature for $1-8 \mathrm{~h}$ and then heated at reflux until the reaction solution clarified. After the solvent was evaporated under atmospheric pressure, the residual liquid could be evaporated under reduced pressure.

\section{Phenyl isocyanate (2a)}

To a solution of bis(trichloromethyl) carbonate $(16.3 \mathrm{~g}, 0.55 \mathrm{~mol})$ in $\mathrm{CHCl}_{3}$ $(60 \mathrm{ml})$ was added dropwise $1 \mathbf{a}(9.3 \mathrm{~g}, 0.1 \mathrm{~mol})$ in $\mathrm{CHCl}_{3}(40 \mathrm{ml})$ in an ice bath. When the addition was finished, the reaction mixture was stirred at room temperature for $1 \mathrm{~h}$ and then heated at reflux until the reaction solution clarified. After the solvent was evaporated under atmospheric pressure, the residual liquid was evaporated under reduced pressure to give $10.1 \mathrm{~g}(85 \%)$ of 2a as colorless liquid: boiling point (bp) $65-68^{\circ} \mathrm{C}$ per $30 \mathrm{~mm} \mathrm{Hg}$.

\section{4-Methylphenyl isocyanate (2b)}

$1 \mathbf{b}(10.8 \mathrm{~g}, 0.1 \mathrm{~mol})$ in $\mathrm{CHCl}_{3}$ was added dropwise according to the method for phenyl isocyanate. When the addition was finished, the reaction mixture was stirred at room temperature for $2 \mathrm{~h}$. The solvent was evaporated to give $14.04 \mathrm{~g}$ (90\%) of $\mathbf{2 b}$ as colorless liquid: bp $69-70{ }^{\circ} \mathrm{C}$ per $30 \mathrm{~mm} \mathrm{Hg}$.
4-Methoxyphenyl isocyanate (2c)

1c $(12.4 \mathrm{~g}, 0.1 \mathrm{~mol})$ in $\mathrm{CHCl}_{3}$ was added dropwise according to the method for phenyl isocyanate. When the addition was finished, the reaction mixture was stirred at room temperature for $1 \mathrm{~h}$. The solvent was evaporated to give $12.75 \mathrm{~g}$ (85\%) of $2 \mathrm{c}$ as colorless liquid: bp $75-77^{\circ} \mathrm{C}$ per $30 \mathrm{~mm} \mathrm{Hg}$.

\section{4-Nitrophenyl isocyanate (2d)}

1d $(13.8 \mathrm{~g}, 0.1 \mathrm{~mol})$ in $\mathrm{CHCl}_{3}$ was added dropwise according to the method for phenyl isocyanate. When the addition was finished, the reaction mixture was stirred at room temperature for $3 \mathrm{~h}$. The solvent was evaporated to give $13.27 \mathrm{~g}$ (81\%) of $\mathbf{2 d}$ as yellow solid: bp $85-86^{\circ} \mathrm{C}$ per $30 \mathrm{~mm} \mathrm{Hg}$.

\section{3-Methylphenyl isocyanate (2e)}

le $(10.8 \mathrm{~g}, 0.1 \mathrm{~mol})$ in $\mathrm{CHCl}_{3}$ was added dropwise according to the method for phenyl isocyanate. When the addition was finished, the reaction mixture was stirred at room temperature for $3 \mathrm{~h}$. The solvent was evaporated to give $11.13 \mathrm{~g}$ (70\%) of $2 \mathrm{e}$ as colorless liquid: bp $69-70{ }^{\circ} \mathrm{C}$ per $30 \mathrm{~mm} \mathrm{Hg}$.

\section{2-Chlorophenyl isocyanate (2f)}

If $(12.85 \mathrm{~g}, 0.1 \mathrm{~mol})$ in $\mathrm{CHCl}_{3}$ was added dropwise according to the method for phenyl isocyanate. When the addition was finished, the reaction mixture was stirred at room temperature for $1.5 \mathrm{~h}$. The solvent was evaporated to give $11.87 \mathrm{~g}(77 \%)$ of $\mathbf{2 f}$ as colorless liquid: bp $64-66{ }^{\circ} \mathrm{C}$ per $20 \mathrm{~mm} \mathrm{Hg}$.

\section{3-Chlorophenyl isocyanate (2g)}

$1 \mathrm{~g}(12.86 \mathrm{~g}, 0.1 \mathrm{~mol})$ in $\mathrm{CHCl}_{3}$ was added dropwise according to the method for phenyl isocyanate. When the addition was finished, the reaction mixture was stirred at room temperature for $3.5 \mathrm{~h}$. The solvent was evaporated to give $11.29 \mathrm{~g}(73 \%)$ of $2 \mathrm{~g}$ as colorless liquid: bp $66-68^{\circ} \mathrm{C}$ per $20 \mathrm{~mm} \mathrm{Hg}$.

\section{4-Trifluoromethylphenyl isocyanate $(2 \mathrm{~h})$}

$1 \mathbf{h}(16.1 \mathrm{~g}, 0.1 \mathrm{~mol})$ in $\mathrm{CHCl}_{3}$ was added dropwise according to the method for phenyl isocyanate. When the addition was finished, the reaction mixture was stirred at room temperature for $3 \mathrm{~h}$. The solvent was evaporated to give $15.01 \mathrm{~g}$ (80\%) of $\mathbf{2 h}$ as colorless liquid: bp $71-73^{\circ} \mathrm{C}$ per $30 \mathrm{~mm} \mathrm{Hg}$.

\section{2'-O-Benzoyl-11-deoxy-3-des(hexopyranosyloxy)-11-(3-} hydroxyprolylamino)-6-O-methyl-3-oxoerythromycin A 11-, $12-\mathrm{O}-$ cyclic carbamate (4)

2'-O-Benzoyl-10,11-dehydro-3-des(hexopyranosyloxy)-12-O-imidazolylcarbonyl6-O-methyl-3-oxoerythromycin A (3) $(10.7 \mathrm{~g}, 13.9 \mathrm{mmol})$ and 1-amino propanol $(11 \mathrm{ml}, 14.3 \mathrm{mmol})$ were dissolved in DMF $(80 \mathrm{ml})$ and the mixture was stirred for $62 \mathrm{~h}$ at room temperature. Water $(150 \mathrm{ml})$ was added to the resulting solution and the aqueous layer was extracted with ethyl acetate $(3 \times 100 \mathrm{ml})$. The combined organic layers were washed with water $(150 \mathrm{ml})$, brine $(100 \mathrm{ml})$, dried over anhydrous $\mathrm{Na}_{2} \mathrm{SO}_{4}$, filtered and concentrated in vacuum. The residue was purified by flash chromatography (acetone:hexane, 3:6) to give $10.7 \mathrm{~g}(85 \%)$ of $\mathbf{4}$ as a white solid.

${ }^{1} \mathrm{H} \quad \mathrm{NMR}\left(\mathrm{CDCl}_{3}, 400 \mathrm{MHz}\right) \quad \delta \quad 8.05(\mathrm{~d}, J=7.6 \mathrm{~Hz}, 2 \mathrm{H}$, phenyl- $\mathrm{H} 2$, phenyl-H6), $7.63(\mathrm{t}, J=6.9 \mathrm{~Hz}, 1 \mathrm{H}$, phenyl- $H 4), 7.49(\mathrm{t}, J=7.6 \mathrm{~Hz}, 2 \mathrm{H}$, phenyl- $H 3$, phenyl- $H 5), 4.92(\mathrm{dd}, J=2.1,8.7 \mathrm{~Hz}, 1 \mathrm{H}, 13-H), 4.57(\mathrm{dd}, J=8.0$, $\left.10.1 \mathrm{~Hz}, 1 \mathrm{H}, 2^{\prime}-H\right), 4.12(\mathrm{~d}, 1 \mathrm{H}, J=10.8 \mathrm{~Hz}, 5-H), 3.83(\mathrm{q}, J=7.8 \mathrm{~Hz}, 1 \mathrm{H}, 2-H)$, $3.72\left(\mathrm{~m}, 2 \mathrm{H}, \mathrm{CH}_{2} \mathrm{CH}_{2} \mathrm{CH}_{2} \mathrm{OH}\right), 3.55(\mathrm{~d}, J=2.5 \mathrm{~Hz}, 1 \mathrm{H}, 11-\mathrm{H}), 3.50(\mathrm{~m}, 1 \mathrm{H}$, $\left.5^{\prime}-H\right), 3.41(\mathrm{~m}, 1 \mathrm{H}, 4-H), 3.10(\mathrm{q}, J=7.0 \mathrm{~Hz}, 1 \mathrm{H}, 8-H), 3.05(\mathrm{ql}, J=6.9 \mathrm{~Hz}, 1 \mathrm{H}$, $10-\mathrm{H}), 2.86\left(\mathrm{~m}, 2 \mathrm{H}, \mathrm{NCH}_{2} \mathrm{CH}_{2} \mathrm{CH}_{2} \mathrm{OH}\right), 2.78\left(\mathrm{~s}, 3 \mathrm{H}, 6-\mathrm{O}-\mathrm{CH}_{3}\right), 2.7(\mathrm{~m}, 1 \mathrm{H}$, $\left.3^{\prime}-\mathrm{H}\right), 2.26\left(\mathrm{~s}, 6 \mathrm{H}, \mathrm{N}\left(\mathrm{CH}_{3}\right)_{2}\right), 1.93(\mathrm{~m}, 1 \mathrm{H}, 14-\mathrm{Ha}), 1.73\left(\mathrm{~m}, 1 \mathrm{H}, 4^{\prime}-\mathrm{Ha}\right)$, $1.65\left(\mathrm{~m}, 2 \mathrm{H}, \mathrm{NCH}_{2} \mathrm{CH}_{2} \mathrm{CH}_{2} \mathrm{OH}\right), 1.63(\mathrm{~m}, 1 \mathrm{H}, 14-\mathrm{Hb}), 1.52\left(\mathrm{~s}, 3 \mathrm{H}, 6-\mathrm{CH}_{3}\right)$, $1.38(\mathrm{~m}, 1 \mathrm{H}, 7-\mathrm{Ha}), 1.32(\mathrm{~m}, 1 \mathrm{H}, 7-\mathrm{Hb}), 1.26\left(\mathrm{~s}, 3 \mathrm{H}, 12-\mathrm{CH}_{3}\right), 1.24(\mathrm{~m}, 1 \mathrm{H}$, $\left.4^{\prime}-\mathrm{Hb}\right), 1.17\left(\mathrm{~d}, J=7.0 \mathrm{~Hz}, 3 \mathrm{H}, 8-\mathrm{CH}_{3}\right), 1.13\left(\mathrm{~d}, J=7.8 \mathrm{~Hz}, 3 \mathrm{H}, 2-\mathrm{CH}_{3}\right)$, $1.12\left(\mathrm{~d}, \quad J=6.9 \mathrm{~Hz}, \quad 3 \mathrm{H}, \quad 10-\mathrm{CH}_{3}\right), \quad 1.10\left(\mathrm{~d}, \quad J=7.4 \mathrm{~Hz}, \quad 3 \mathrm{H}, \quad 4-\mathrm{CH}_{3}\right)$, $0.80(\mathrm{t}, J=7.2 \mathrm{~Hz}, 3 \mathrm{H}, 15-\mathrm{H})$. ESI-MS $(\mathrm{m} / \mathrm{z}): 775(\mathrm{M}+\mathrm{H})^{+}$. 
General methods for 11-[3-[(arylcarbamoyl)oxy]propylamino]-2'O-benzoyl-11-deoxy-3-des(hexopyranosyloxy)-6-O-methyl-3oxoerythromycin A 11- $\mathrm{N}, 12-\mathrm{O}$-cyclic carbamates (5a-h)

To a solution of 2'-O-benzoyl-11-deoxy-3-des(hexopyranosyloxy)-11(3-hydroxyprolylamino)-6-O-methyl-3-oxoerythromycin A 11-N,12-O-cyclic carbamate (4) $(0.4 \mathrm{~g}, 0.516 \mathrm{mmol})$, DCC $(0.11 \mathrm{~g}, 0.516 \mathrm{mmol})$ and DMAP $(0.063 \mathrm{~g}, 0.516 \mathrm{mmol})$ in $\mathrm{CH}_{2} \mathrm{Cl}_{2}(20 \mathrm{ml})$ was added dropwise corresponding aryl isocyanate $(2.58 \mathrm{mmol})$ in $\mathrm{CH}_{2} \mathrm{Cl}_{2}(10 \mathrm{ml})$ in an ice bath. The reaction mixture was allowed to slowly warm to room temperature and was stirred for $20 \mathrm{~h}$ under nitrogen. The reaction was quenched with methanol $(3 \mathrm{ml})$, filtered and concentrated in vacuum. The residue was purified by flash chromatography (cyclohexane-ethyl acetate, 5:1 2:1) to afford products $\mathbf{5 a - h}$ as a white solid.

2'-O-Benzoyl-11-deoxy-3-des(hexopyranosyloxy)-6-O-methyl-3oxo-11-[3-[(phenylcarbamoyl)oxy]propylamino]erythromycin A $11-\mathrm{N}, 12-\mathrm{O}$-cyclic carbamate $(5 \mathrm{a})$

White solid, yield $82 \% .{ }^{1} \mathrm{H}$ NMR $\left(\mathrm{CDCl}_{3}, 400 \mathrm{MHz}\right) \delta 8.08(\mathrm{~d}, J=7.8 \mathrm{~Hz}, 2 \mathrm{H}$, phenyl- $H 2$, phenyl- $H 6), 7.68(\mathrm{t}, J=6.9 \mathrm{~Hz}, 1 \mathrm{H}$, phenyl- $H 4), 7.49(\mathrm{t}, J=6.0 \mathrm{~Hz}$, $2 \mathrm{H}$, phenyl- $H 3$, phenyl- $H 5), 7.38(\mathrm{~d}, J=8.0 \mathrm{~Hz}, 2 \mathrm{H}$, phenyl- $H 2$, phenyl- $H 6)$, $7.28(\mathrm{~d}, J=8.0 \mathrm{~Hz}, 2 \mathrm{H}$, phenyl- $H 3$, phenyl- $H 5), 7.05(\mathrm{t}, J=7.6 \mathrm{~Hz}, 1 \mathrm{H}$, phenyl$H 4$ ), 4.92 (dd, $J=2.1,8.7 \mathrm{~Hz}, 1 \mathrm{H}, 13-H), 4.57\left(\mathrm{dd}, J=8.0,10.1 \mathrm{~Hz}, 1 \mathrm{H}, 2^{\prime}-H\right.$ ), $4.12(\mathrm{~d}, 1 \mathrm{H}, J=10.8 \mathrm{~Hz}, 5-H), 3.83(\mathrm{q}, J=7.8 \mathrm{~Hz}, 1 \mathrm{H}, 2-H), 3.72(\mathrm{~m}, 2 \mathrm{H}$, $\left.\mathrm{CH}_{2} \mathrm{CH}_{2} \mathrm{CH}_{2} \mathrm{OH}\right), \quad 3.55(\mathrm{~d}, J=2.5 \mathrm{~Hz}, 1 \mathrm{H}, \quad 11-\mathrm{H}), 3.50\left(\mathrm{~m}, 1 \mathrm{H}, \quad 5^{\prime}-\mathrm{H}\right)$, $3.41(\mathrm{~m}, 1 \mathrm{H}, 4-H), 3.10(\mathrm{q}, J=7.0 \mathrm{~Hz}, 1 \mathrm{H}, 8-H), 3.05(\mathrm{ql}, J=6.9 \mathrm{~Hz}, 1 \mathrm{H}$, $10-\mathrm{H}), 2.86\left(\mathrm{~m}, 2 \mathrm{H}, \mathrm{NCH}_{2} \mathrm{CH}_{2} \mathrm{CH}_{2} \mathrm{OH}\right), 2.78\left(\mathrm{~s}, 3 \mathrm{H}, 6-\mathrm{O}_{-} \mathrm{CH}_{3}\right), 2.7(\mathrm{~m}, 1 \mathrm{H}$, $\left.3^{\prime}-\mathrm{H}\right), 2.26\left(\mathrm{~s}, 6 \mathrm{H}, \mathrm{N}\left(\mathrm{CH}_{3}\right)_{2}\right), 1.93(\mathrm{~m}, 1 \mathrm{H}, 14-\mathrm{Ha}), 1.73\left(\mathrm{~m}, 1 \mathrm{H}, 4^{\prime}-\mathrm{Ha}\right), 1.65(\mathrm{~m}$, $\left.2 \mathrm{H}, \mathrm{NCH}_{2} \mathrm{CH}_{2} \mathrm{CH}_{2} \mathrm{OH}\right), 1.63(\mathrm{~m}, 1 \mathrm{H}, 14-\mathrm{Hb}), 1.52\left(\mathrm{~s}, 3 \mathrm{H}, 6-\mathrm{CH}_{3}\right), 1.38(\mathrm{~m}, 1 \mathrm{H}$, $7-\mathrm{Ha}), 1.32(\mathrm{~m}, 1 \mathrm{H}, 7-\mathrm{Hb}), 1.26\left(\mathrm{~s}, 3 \mathrm{H}, 12-\mathrm{CH}_{3}\right), 1.24\left(\mathrm{~m}, 1 \mathrm{H}, 4^{\prime}-\mathrm{Hb}\right), 1.17$ $\left(\mathrm{d}, J=7.0 \mathrm{~Hz}, 3 \mathrm{H}, 8-\mathrm{CH}_{3}\right), 1.13\left(\mathrm{~d}, J=7.8 \mathrm{~Hz}, 3 \mathrm{H}, 2-\mathrm{CH}_{3}\right), 1.12(\mathrm{~d}, J=6.9 \mathrm{~Hz}$, $\left.3 \mathrm{H}, 10-\mathrm{CH}_{3}\right), 1.10\left(\mathrm{~d}, J=7.4 \mathrm{~Hz}, 3 \mathrm{H}, 4-\mathrm{CH}_{3}\right), 0.78(\mathrm{t}, J=7.2 \mathrm{~Hz}, 3 \mathrm{H}, 15-\mathrm{H})$. $\operatorname{ESI-MS}(m / z): 894(\mathrm{M}+\mathrm{H})^{+}$.

\section{2'-O-Benzoyl-11-deoxy-3-des(hexopyranosyloxy)-6-O-methyl-11-} [3-[(4-methylphenylcarbamoyl)oxy]propylamino]-3-oxoerythromycin A 11- N,12-O-cyclic carbamate (5b)

White solid, yield $85 \% .{ }^{1} \mathrm{H}$ NMR $\left(\mathrm{CDCl}_{3}, 400 \mathrm{MHz}\right) \delta 8.08(\mathrm{~d}, J=7.8 \mathrm{~Hz}, 2 \mathrm{H}$, phenyl- $H 2$, phenyl-H6), $7.68(\mathrm{t}, J=6.9 \mathrm{~Hz}, 1 \mathrm{H}$, phenyl- $H 4), 7.49(\mathrm{t}, J=6.0 \mathrm{~Hz}$, $2 \mathrm{H}$, phenyl- $H 3$, phenyl- $H 5), 7.37(\mathrm{~d}, J=8.0 \mathrm{~Hz}, 2 \mathrm{H}$, phenyl- $H 2$, phenyl- $H 6$ ), $7.06(\mathrm{~d}, J=8.0 \mathrm{~Hz}, 2 \mathrm{H}$, phenyl- $\mathrm{H}$, phenyl- $H 5), 4.92(\mathrm{dd}, J=2.1,8.7 \mathrm{~Hz}, 1 \mathrm{H}$, $13-H), 4.57\left(\mathrm{dd}, J=8.0,10.1 \mathrm{~Hz}, 1 \mathrm{H}, 2^{\prime}-H\right), 4.12(\mathrm{~d}, 1 \mathrm{H}, J=10.8 \mathrm{~Hz}, 5-H), 3.83$ (q, $J=7.8 \mathrm{~Hz}, 1 \mathrm{H}, 2-\mathrm{H}), 3.72\left(\mathrm{~m}, 2 \mathrm{H}, \mathrm{CH}_{2} \mathrm{CH}_{2} \mathrm{CH}_{2} \mathrm{OH}\right), 3.55(\mathrm{~d}, J=2.5 \mathrm{~Hz}, 1 \mathrm{H}$, $11-H), 3.50\left(\mathrm{~m}, 1 \mathrm{H}, 5^{\prime}-H\right), 3.41(\mathrm{~m}, 1 \mathrm{H}, 4-H), 3.10(\mathrm{q}, J=7.0 \mathrm{~Hz}, 1 \mathrm{H}, 8-H), 3.05$ $(\mathrm{ql}, J=6.9 \mathrm{~Hz}, 1 \mathrm{H}, 10-\mathrm{H}), 2.86\left(\mathrm{~m}, 2 \mathrm{H}, \mathrm{NCH}_{2} \mathrm{CH}_{2} \mathrm{CH}_{2} \mathrm{OH}\right), 2.78(\mathrm{~s}, 3 \mathrm{H}$, 6- $\left.-\mathrm{CH}_{3}\right), 2.7\left(\mathrm{~m}, 1 \mathrm{H}, 3^{\prime}-\mathrm{H}\right), 2.27\left(\mathrm{~s}, 6 \mathrm{H}, \mathrm{N}\left(\mathrm{CH}_{3}\right)_{2}\right), 2.24\left(\mathrm{~s}, 3 \mathrm{H}\right.$, phenyl- $\left.\mathrm{CH}_{3}\right)$, $1.93(\mathrm{~m}, 1 \mathrm{H}, 14-\mathrm{Ha}), 1.73\left(\mathrm{~m}, 1 \mathrm{H}, 4^{\prime}-\mathrm{Ha}\right), 1.65\left(\mathrm{~m}, 2 \mathrm{H}, \mathrm{NCH}_{2} \mathrm{CH}_{2} \mathrm{CH}_{2} \mathrm{OH}\right)$, $1.63(\mathrm{~m}, 1 \mathrm{H}, 14-\mathrm{Hb}), 1.52\left(\mathrm{~s}, 3 \mathrm{H}, 6-\mathrm{CH}_{3}\right), 1.38(\mathrm{~m}, 1 \mathrm{H}, 7-\mathrm{Hb}), 1.32(\mathrm{~m}, 1 \mathrm{H}$, $7-\mathrm{Hb}), 1.26\left(\mathrm{~s}, 3 \mathrm{H}, 12-\mathrm{CH}_{3}\right), 1.24\left(\mathrm{~m}, 1 \mathrm{H}, 4^{\prime}-\mathrm{Hb}\right), 1.17(\mathrm{~d}, J=7.0 \mathrm{~Hz}, 3 \mathrm{H}$, $\left.8-\mathrm{CH}_{3}\right), 1.13\left(\mathrm{~d}, J=7.8 \mathrm{~Hz}, 3 \mathrm{H}, 2-\mathrm{CH}_{3}\right), 1.12\left(\mathrm{~d}, J=6.9 \mathrm{~Hz}, 3 \mathrm{H}, 10-\mathrm{CH}_{3}\right)$, $1.10\left(\mathrm{~d}, J=7.4 \mathrm{~Hz}, 3 \mathrm{H}, 4-\mathrm{CH}_{3}\right), 0.78(\mathrm{t}, J=7.2 \mathrm{~Hz}, 3 \mathrm{H}, 15-\mathrm{H}) . \operatorname{ESI}-\mathrm{MS}(\mathrm{m} / z)$ : $908(\mathrm{M}+\mathrm{H})^{+}$.

2'-O-Benzoyl-11-deoxy-3-des(hexopyranosyloxy)-6-O-methyl-11[3-[(4-methoxyphenylcarbamoyl)oxy]propylamino]-3-oxoerythromycin A 11- N,12-O-cyclic carbamate $(5 \mathrm{c})$

White solid, yield $83 \%$. ${ }^{1} \mathrm{H}$ NMR $\left(\mathrm{CDCl}_{3}, 400 \mathrm{MHz}\right) \delta 8.08(\mathrm{~d}, J=7.8 \mathrm{~Hz}, 2 \mathrm{H}$, phenyl- $H 2$, phenyl-H6), $7.68(\mathrm{t}, J=6.9 \mathrm{~Hz}, 1 \mathrm{H}$, phenyl- $H 4), 7.49(\mathrm{t}, J=6.0 \mathrm{~Hz}$, $2 \mathrm{H}$, phenyl- $H 3$, phenyl- $H 5), 7.47(\mathrm{~d}, J=8.0 \mathrm{~Hz}, 2 \mathrm{H}$, phenyl- $H 2$, phenyl- $H 6$ ), $6.89(\mathrm{~d}, J=8.0 \mathrm{~Hz}, 2 \mathrm{H}$, phenyl- $H 3$, phenyl- $H 5), 4.92(\mathrm{dd}, J=2.1,8.7 \mathrm{~Hz}, 1 \mathrm{H}$, $13-H), 4.57\left(\mathrm{dd}, J=8.0,10.1 \mathrm{~Hz}, 1 \mathrm{H}, 2^{\prime}-H\right), 4.12(\mathrm{~d}, 1 \mathrm{H}, J=10.8 \mathrm{~Hz}, 5-H), 3.83$ $(\mathrm{q}, J=7.8 \mathrm{~Hz}, \quad 1 \mathrm{H}, \quad 2-\mathrm{H}), \quad 3.72\left(\mathrm{~m}, \quad 2 \mathrm{H}, \mathrm{CH}_{2} \mathrm{CH}_{2} \mathrm{CH}_{2} \mathrm{OH}\right), 3.60(\mathrm{~s}, \quad 3 \mathrm{H}$, phenyl- $\left.-\mathrm{OCH}_{3}\right), \quad 3.55(\mathrm{~d}, J=2.5 \mathrm{~Hz}, 1 \mathrm{H}, \quad 11-\mathrm{H}), \quad 3.50\left(\mathrm{~m}, 1 \mathrm{H}, 5^{\prime}-\mathrm{H}\right), 3.41$ $(\mathrm{m}, 1 \mathrm{H}, 4-H), 3.10(\mathrm{q}, J=7.0 \mathrm{~Hz}, 1 \mathrm{H}, 8-H), 3.05(\mathrm{ql}, J=6.9 \mathrm{~Hz}, 1 \mathrm{H}, 10-H)$, 2.86(m, $\left.2 \mathrm{H}, \mathrm{NCH}_{2} \mathrm{CH}_{2} \mathrm{CH}_{2} \mathrm{OH}\right), 2.78\left(\mathrm{~s}, 3 \mathrm{H}, 6-\mathrm{O}-\mathrm{CH}_{3}\right), 2.7\left(\mathrm{~m}, 1 \mathrm{H}, 3^{\prime}-\mathrm{H}\right), 2.27$ (s, $\left.6 \mathrm{H}, \mathrm{N}\left(\mathrm{CH}_{3}\right)_{2}\right), 2.24\left(\mathrm{~s}, 3 \mathrm{H}\right.$, phenyl- $\left.\mathrm{CH}_{3}\right), 1.93(\mathrm{~m}, 1 \mathrm{H}, 14-\mathrm{Ha}), 1.73(\mathrm{~m}, 1 \mathrm{H}$, $\left.4^{\prime}-\mathrm{Ha}\right), 1.65\left(\mathrm{~m}, 2 \mathrm{H}, \mathrm{NCH}_{2} \mathrm{CH}_{2} \mathrm{CH}_{2} \mathrm{OH}\right), 1.63(\mathrm{~m}, 1 \mathrm{H}, 14-\mathrm{Hb}), 1.52(\mathrm{~s}, 3 \mathrm{H}$,
6- $\left.\mathrm{CH}_{3}\right), 1.38(\mathrm{~m}, 1 \mathrm{H}, 7-\mathrm{Ha}), 1.32(\mathrm{~m}, 1 \mathrm{H}, 7-\mathrm{Hb}), 1.26\left(\mathrm{~s}, 3 \mathrm{H}, 12-\mathrm{CH}_{3}\right), 1.24$ $\left(\mathrm{m}, 1 \mathrm{H}, 4^{\prime}-\mathrm{Hb}\right), 1.17\left(\mathrm{~d}, J=7.0 \mathrm{~Hz}, 3 \mathrm{H}, 8-\mathrm{CH}_{3}\right), 1.13\left(\mathrm{~d}, J=7.8 \mathrm{~Hz}, 3 \mathrm{H}, 2-\mathrm{CH}_{3}\right)$, $1.12\left(\mathrm{~d}, \quad J=6.9 \mathrm{~Hz}, \quad 3 \mathrm{H}, \quad 10-\mathrm{CH}_{3}\right), \quad 1.10\left(\mathrm{~d}, \quad J=7.4 \mathrm{~Hz}, \quad 3 \mathrm{H}, \quad 4-\mathrm{CH}_{3}\right)$, $0.78(\mathrm{t}, J=7.2 \mathrm{~Hz}, 3 \mathrm{H}, 15-H)$. ESI-MS $(\mathrm{m} / \mathrm{z}): 924(\mathrm{M}+\mathrm{H})^{+}$.

\section{2'-O-Benzoyl-11-deoxy-3-des(hexopyranosyloxy)-6-O-methyl-11-} [3-[(4-nitrophenylcarbamoyl)oxy]propylamino]-3-oxoerythromycin A 11-N,12-O-cyclic carbamate (5d)

White solid, yield $78 \% .{ }^{1} \mathrm{H}$ NMR $\left(\mathrm{CDCl}_{3}, 400 \mathrm{MHz}\right) \delta 8.08(\mathrm{~d}, J=7.8 \mathrm{~Hz}, 2 \mathrm{H}$, phenyl- $H 2$, phenyl- $H 6$ ), $8.05(\mathrm{~d}, J=8.2 \mathrm{~Hz}, 2 \mathrm{H}$, phenyl- $H 2$, phenyl- $H 6), 7.68$ (t, $J=6.9 \mathrm{~Hz}, 1 \mathrm{H}$, phenyl- $H 4), 7.63(\mathrm{~d}, J=8.2 \mathrm{~Hz}, 2 \mathrm{H}$, phenyl- $H 3$, phenyl- $H 5)$, $7.49(\mathrm{t}, J=6.0 \mathrm{~Hz}, 2 \mathrm{H}$, phenyl- $H 3$, phenyl- $H 5), 4.92(\mathrm{dd}, J=2.1,8.7 \mathrm{~Hz}, 1 \mathrm{H}$, $13-H), 4.57\left(\mathrm{dd}, J=8.0,10.1 \mathrm{~Hz}, 1 \mathrm{H}, 2^{\prime}-H\right), 4.12(\mathrm{~d}, J=10.8 \mathrm{~Hz}, 1 \mathrm{H}, 5-H), 3.83$ (q, $J=7.8 \mathrm{~Hz}, \quad 1 \mathrm{H}, 2-\mathrm{H}), \quad 3.72\left(\mathrm{~m}, 2 \mathrm{H}, \quad \mathrm{CH}_{2} \mathrm{CH}_{2} \mathrm{CH}_{2} \mathrm{OH}\right), 3.60(\mathrm{~s}, \quad 3 \mathrm{H}$, phenyl- $\left.-\mathrm{OCH}_{3}\right), \quad 3.55(\mathrm{~d}, J=2.5 \mathrm{~Hz}, 1 \mathrm{H}, 11-\mathrm{H}), \quad 3.50\left(\mathrm{~m}, 1 \mathrm{H}, 5^{\prime}-\mathrm{H}\right), 3.41$ $(\mathrm{m}, 1 \mathrm{H}, 4-H), 3.10(\mathrm{q}, J=7.0 \mathrm{~Hz}, 1 \mathrm{H}, 8-H), 3.05(\mathrm{ql}, J=6.9 \mathrm{~Hz}, 1 \mathrm{H}, 10-H)$, $2.86\left(\mathrm{~m}, 2 \mathrm{H}, \mathrm{NCH}_{2} \mathrm{CH}_{2} \mathrm{CH}_{2} \mathrm{OH}\right), 2.78\left(\mathrm{~s}, 3 \mathrm{H}, 6-\mathrm{O}-\mathrm{CH}_{3}\right), 2.7\left(\mathrm{~m}, 1 \mathrm{H}, 3^{\prime}-\mathrm{H}\right), 2.27$ $\left(\mathrm{s}, 6 \mathrm{H}, \mathrm{N}\left(\mathrm{CH}_{3}\right)_{2}\right), 2.24\left(\mathrm{~s}, 3 \mathrm{H}\right.$, phenyl- $\left.\mathrm{CH}_{3}\right), 1.93(\mathrm{~m}, 1 \mathrm{H}, 14-\mathrm{Ha}), 1.73(\mathrm{~m}, 1 \mathrm{H}$, $\left.4^{\prime}-\mathrm{Ha}\right), 1.65\left(\mathrm{~m}, 2 \mathrm{H}, \mathrm{NCH}_{2} \mathrm{CH}_{2} \mathrm{CH}_{2} \mathrm{OH}\right), 1.63(\mathrm{~m}, 1 \mathrm{H}, 14-\mathrm{Hb}), 1.52(\mathrm{~s}, 3 \mathrm{H}$, $\left.6-\mathrm{CH}_{3}\right), 1.38(\mathrm{~m}, 1 \mathrm{H}, 7-\mathrm{Ha}), 1.32(\mathrm{~m}, 1 \mathrm{H}, 7-\mathrm{Hb}), 1.26\left(\mathrm{~s}, 3 \mathrm{H}, 12-\mathrm{CH}_{3}\right)$, $1.24\left(\mathrm{~m}, 1 \mathrm{H}, 4^{\prime}-\mathrm{Hb}\right), 1.17\left(\mathrm{~d}, J=7.0 \mathrm{~Hz}, 3 \mathrm{H}, 8-\mathrm{CH}_{3}\right), 1.13(\mathrm{~d}, J=7.8 \mathrm{~Hz}$, $\left.3 \mathrm{H}, 2-\mathrm{CH}_{3}\right), 1.12\left(\mathrm{~d}, J=6.9 \mathrm{~Hz}, 3 \mathrm{H}, 10-\mathrm{CH}_{3}\right), 1.10\left(\mathrm{~d}, J=7.4 \mathrm{~Hz}, 3 \mathrm{H}, 4-\mathrm{CH}_{3}\right)$, $0.78(\mathrm{t}, J=7.2 \mathrm{~Hz}, 3 \mathrm{H}, 15-\mathrm{H})$. ESI-MS $(m / z): 939(\mathrm{M}+\mathrm{H})^{+}$.

\section{2'-O-Benzoyl-11-deoxy-3-des(hexopyranosyloxy)-6-O-methyl-11-} [3-[(3-methylphenylcarbamoyl)oxy]propylamino]-3-oxoerythromycin A 11- N,12-O-cyclic carbamate (5e)

White solid, yield $85 \% .{ }^{1} \mathrm{H}$ NMR $\left(\mathrm{CDCl}_{3}, 400 \mathrm{MHz}\right) \delta 8.08(\mathrm{~d}, J=7.8 \mathrm{~Hz}, 2 \mathrm{H}$, phenyl-H2, phenyl-H6), $7.68(\mathrm{t}, J=6.9 \mathrm{~Hz}, 1 \mathrm{H}$, phenyl-H4), $7.50(\mathrm{~s}, 1 \mathrm{H}$, phenyl-H2), $\quad 7.49(\mathrm{t}, \quad J=6.0 \mathrm{~Hz}, \quad 2 \mathrm{H}, \quad$ phenyl- $H 3$, phenyl- $H 5), \quad 7.45(\mathrm{~d}$, $J=7.7 \mathrm{~Hz}, \quad 1 \mathrm{H}$, phenyl-H6), $7.04(\mathrm{~d}, J=7.7 \mathrm{~Hz}, 1 \mathrm{H}$, phenyl-H5), $6.82(\mathrm{~d}$, $J=7.7 \mathrm{~Hz}, 1 \mathrm{H}$, phenyl-H4), $4.92(\mathrm{dd}, J=2.1,8.7 \mathrm{~Hz}, 1 \mathrm{H}, 13-H), 4.57(\mathrm{dd}$, $\left.J=8.0,10.1 \mathrm{~Hz}, 1 \mathrm{H}, 2^{\prime}-H\right), 4.12(\mathrm{~d}, 1 \mathrm{H}, J=10.8 \mathrm{~Hz}, 5-H), 3.83(\mathrm{q}, J=7.8 \mathrm{~Hz}$, $1 \mathrm{H}, 2-\mathrm{H}), 3.72\left(\mathrm{~m}, 2 \mathrm{H}, \mathrm{CH}_{2} \mathrm{CH}_{2} \mathrm{CH}_{2} \mathrm{OH}\right), 3.55(\mathrm{~d}, J=2.5 \mathrm{~Hz}, 1 \mathrm{H}, 11-\mathrm{H}), 3.50$ $\left(\mathrm{m}, 1 \mathrm{H}, \quad 5^{\prime}-H\right), 3.41(\mathrm{~m}, 1 \mathrm{H}, 4-H), 3.10(\mathrm{q}, J=7.0 \mathrm{~Hz}, 1 \mathrm{H}, 8-\mathrm{H}), 3.05$ $(\mathrm{ql}, J=6.9 \mathrm{~Hz}, 1 \mathrm{H}, 10-\mathrm{H}), 2.86\left(\mathrm{~m}, 2 \mathrm{H}, \mathrm{NCH}_{2} \mathrm{CH}_{2} \mathrm{CH}_{2} \mathrm{OH}\right), 2.78(\mathrm{~s}, 3 \mathrm{H}$, 6-O- $\left.\mathrm{CH}_{3}\right), 2.7\left(\mathrm{~m}, 1 \mathrm{H}, 3^{\prime}-\mathrm{H}\right), 2.27\left(\mathrm{~s}, 6 \mathrm{H}, \mathrm{N}\left(\mathrm{CH}_{3}\right)_{2}\right), 2.24\left(\mathrm{~s}, 3 \mathrm{H}\right.$, phenyl- $\left.\mathrm{CH}_{3}\right)$, $1.93(\mathrm{~m}, 1 \mathrm{H}, 14-\mathrm{Ha}), 1.73\left(\mathrm{~m}, 1 \mathrm{H}, 4^{\prime}-\mathrm{Ha}\right), 1.65\left(\mathrm{~m}, 2 \mathrm{H}, \mathrm{NCH}_{2} \mathrm{CH}_{2} \mathrm{CH}_{2} \mathrm{OH}\right)$, $1.63(\mathrm{~m}, 1 \mathrm{H}, 14-\mathrm{Hb}), 1.52\left(\mathrm{~s}, 3 \mathrm{H}, 6-\mathrm{CH}_{3}\right), 1.38(\mathrm{~m}, 1 \mathrm{H}, 7-\mathrm{Ha}), 1.32(\mathrm{~m}, 1 \mathrm{H}$, $7-\mathrm{Hb}), 1.26\left(\mathrm{~s}, 3 \mathrm{H}, 12-\mathrm{CH}_{3}\right), 1.24\left(\mathrm{~m}, 1 \mathrm{H}, 4^{\prime}-\mathrm{Hb}\right), 1.17(\mathrm{~d}, J=7.0 \mathrm{~Hz}, 3 \mathrm{H}$, $\left.8-\mathrm{CH}_{3}\right), 1.13\left(\mathrm{~d}, J=7.8 \mathrm{~Hz}, 3 \mathrm{H}, 2-\mathrm{CH}_{3}\right), 1.12\left(\mathrm{~d}, J=6.9 \mathrm{~Hz}, 3 \mathrm{H}, 10-\mathrm{CH}_{3}\right)$, $1.10\left(\mathrm{~d}, J=7.4 \mathrm{~Hz}, 3 \mathrm{H}, 4-\mathrm{CH}_{3}\right), 0.78(\mathrm{t}, J=7.2 \mathrm{~Hz}, 3 \mathrm{H}, 15-\mathrm{H})$. ESI-MS $(m / z)$ : $908(\mathrm{M}+\mathrm{H})^{+}$.

2'-O-Benzoyl-11-[3-[(2-chlorophenylcarbamoyl)oxy]propylamino]11-deoxy-3-des(hexopyranosyloxy)-6-O-methyl-3-oxoerythromycin A 11-, $12-O$-cyclic carbamate (5f)

White solid, yield $78 \% .{ }^{1} \mathrm{H}$ NMR $\left(\mathrm{CDCl}_{3}, 400 \mathrm{MHz}\right) \delta 8.10(\mathrm{t}, J=6.9 \mathrm{~Hz}$, $1 \mathrm{H}$, phenyl-H6), $8.08(\mathrm{~d}, J=7.8 \mathrm{~Hz}, 2 \mathrm{H}$, phenyl- $\mathrm{H} 2$, phenyl- $H 6), 7.85(\mathrm{~s}, 1 \mathrm{H}$, phenyl-H2), $7.68(\mathrm{t}, J=6.9 \mathrm{~Hz}, 1 \mathrm{H}$, phenyl-H4) $7.49(\mathrm{t}, J=6.0 \mathrm{~Hz}, 2 \mathrm{H}$, phenyl-H3, phenyl-H5), 7.15(t, $J=7.7 \mathrm{~Hz}$, phenyl- $H 3), 7.00(\mathrm{t}, J=8.0 \mathrm{~Hz}$, $1 \mathrm{H}$, phenyl-H5), $6.92(\mathrm{t}, J=8.0 \mathrm{~Hz}, 1 \mathrm{H}$, phenyl-H4), $4.92(\mathrm{dd}, J=2.1,8.7 \mathrm{~Hz}$, $1 \mathrm{H}, 13-H), 4.57\left(\mathrm{dd}, J=8.0,10.1 \mathrm{~Hz}, 1 \mathrm{H}, 2^{\prime}-H\right), 4.12(\mathrm{~d}, J=10.8 \mathrm{~Hz}, 1 \mathrm{H}, 5-H)$, $3.83(\mathrm{q}, J=7.8 \mathrm{~Hz}, 1 \mathrm{H}, 2-\mathrm{H}), 3.72\left(\mathrm{~m}, 2 \mathrm{H}, \mathrm{CH}_{2} \mathrm{CH}_{2} \mathrm{CH}_{2} \mathrm{OH}\right), 3.55(\mathrm{~d}, J=2.5 \mathrm{~Hz}$, $1 \mathrm{H}, 11-\mathrm{H}), 3.50\left(\mathrm{~m}, 1 \mathrm{H}, 5^{\prime}-H\right), 3.41(\mathrm{~m}, 1 \mathrm{H}, 4-H), 3.10(\mathrm{q}, J=7.0 \mathrm{~Hz}, 1 \mathrm{H}, 8-H)$, $3.05(\mathrm{ql}, J=6.9 \mathrm{~Hz}, 1 \mathrm{H}, 10-\mathrm{H}), 2.86\left(\mathrm{~m}, 2 \mathrm{H}, \mathrm{NCH}_{2} \mathrm{CH}_{2} \mathrm{CH}_{2} \mathrm{OH}\right), 2.78(\mathrm{~s}, 3 \mathrm{H}$, 6-O- $\left.\mathrm{CH}_{3}\right), 2.7\left(\mathrm{~m}, 1 \mathrm{H}, 3^{\prime}-\mathrm{H}\right), 2.27\left(\mathrm{~s}, 6 \mathrm{H}, \mathrm{N}\left(\mathrm{CH}_{3}\right)_{2}\right), 2.24\left(\mathrm{~s}, 3 \mathrm{H}\right.$, phenyl- $\left.\mathrm{CH}_{3}\right)$, $1.93(\mathrm{~m}, 1 \mathrm{H}, 14-\mathrm{Ha}), 1.73\left(\mathrm{~m}, 1 \mathrm{H}, 4^{\prime}-\mathrm{Ha}\right), 1.65\left(\mathrm{~m}, 2 \mathrm{H}, \mathrm{NCH}_{2} \mathrm{CH}_{2} \mathrm{CH}_{2} \mathrm{OH}\right)$, $1.63(\mathrm{~m}, 1 \mathrm{H}, 14-\mathrm{Hb}), 1.52\left(\mathrm{~s}, 3 \mathrm{H}, 6-\mathrm{CH}_{3}\right), 1.38(\mathrm{~m}, 1 \mathrm{H}, 7-\mathrm{Ha}), 1.32(\mathrm{~m}, 1 \mathrm{H}$, $7-\mathrm{Hb}), 1.26\left(\mathrm{~s}, 3 \mathrm{H}, 12-\mathrm{CH}_{3}\right), 1.24\left(\mathrm{~m}, 1 \mathrm{H}, 4^{\prime}-\mathrm{Hb}\right), 1.17(\mathrm{~d}, J=7.0 \mathrm{~Hz}, 3 \mathrm{H}$, $\left.8-\mathrm{CH}_{3}\right), 1.13\left(\mathrm{~d}, J=7.8 \mathrm{~Hz}, 3 \mathrm{H}, 2-\mathrm{CH}_{3}\right), 1.12\left(\mathrm{~d}, J=6.9 \mathrm{~Hz}, 3 \mathrm{H}, 10-\mathrm{CH}_{3}\right)$, $1.10\left(\mathrm{~d}, J=7.4 \mathrm{~Hz}, 3 \mathrm{H}, 4-\mathrm{CH}_{3}\right), 0.78(\mathrm{t}, J=7.2 \mathrm{~Hz}, 3 \mathrm{H}, 15-\mathrm{H}) . \operatorname{ESI}-\mathrm{MS}(\mathrm{m} / \mathrm{z})$ : $928(\mathrm{M}+\mathrm{H})^{+}$ 
2'-O-Benzoyl-11-[3-[(3-chlorophenylcarbamoyl)oxy]propylamino]11-deoxy-3-des(hexopyranosyloxy)-6-O-methyl-3-oxoerythromycin A 11- N,12-O-cyclic carbamate (5g)

White solid, yield $75 \% .{ }^{1} \mathrm{H}$ NMR $\left(\mathrm{CDCl}_{3}, 400 \mathrm{MHz}\right) \delta 8.08(\mathrm{~d}, J=7.8 \mathrm{~Hz}, 2 \mathrm{H}$, phenyl- $H 2$, phenyl-H6), $7.85(\mathrm{~s}, 1 \mathrm{H}$, phenyl-H2), $7.68(\mathrm{t}, J=6.9 \mathrm{~Hz}, 1 \mathrm{H}$, phenyl-H4), $7.55(\mathrm{~d}, J=7.7 \mathrm{~Hz}, 1 \mathrm{H}$, phenyl-H6) $7.49(\mathrm{t}, J=6.0 \mathrm{~Hz}, 2 \mathrm{H}$, phenyl-H3, phenyl-H5), $7.21(\mathrm{~d}, \quad J=7.7 \mathrm{~Hz}, \quad 1 \mathrm{H}, \quad$ phenyl-H5), $\quad 7.03$ (d, $J=7.7 \mathrm{~Hz}, \quad 1 \mathrm{H}, \quad$ phenyl- $H 4), \quad 4.92(\mathrm{dd}, J=2.1, \quad 8.7 \mathrm{~Hz}, \quad 1 \mathrm{H}, \quad 13-H)$, $4.57\left(\mathrm{dd}, J=8.0,10.1 \mathrm{~Hz}, 1 \mathrm{H}, 2^{\prime}-H\right), 4.12(\mathrm{~d}, J=10.8 \mathrm{~Hz}, 1 \mathrm{H}, 5-H), 3.83$ (q, $J=7.8 \mathrm{~Hz}, 1 \mathrm{H}, 2-\mathrm{H}), 3.72\left(\mathrm{~m}, 2 \mathrm{H}, \mathrm{CH}_{2} \mathrm{CH}_{2} \mathrm{CH}_{2} \mathrm{OH}\right), 3.55(\mathrm{~d}, J=2.5 \mathrm{~Hz}$, $1 \mathrm{H}, 11-H), 3.50\left(\mathrm{~m}, 1 \mathrm{H}, 5^{\prime}-H\right), 3.41(\mathrm{~m}, 1 \mathrm{H}, 4-H), 3.10(\mathrm{q}, J=7.0 \mathrm{~Hz}, 1 \mathrm{H}, 8-H)$, $3.05(\mathrm{ql}, J=6.9 \mathrm{~Hz}, 1 \mathrm{H}, 10-\mathrm{H}), 2.86\left(\mathrm{~m}, 2 \mathrm{H}, \mathrm{NCH}_{2} \mathrm{CH}_{2} \mathrm{CH}_{2} \mathrm{OH}\right), 2.78(\mathrm{~s}, 3 \mathrm{H}$, 6-O- $\left.\mathrm{CH}_{3}\right), 2.7\left(\mathrm{~m}, 1 \mathrm{H}, 3^{\prime}-\mathrm{H}\right), 2.27\left(\mathrm{~s}, 6 \mathrm{H}, \mathrm{N}\left(\mathrm{CH}_{3}\right)_{2}\right), 2.24\left(\mathrm{~s}, 3 \mathrm{H}\right.$, phenyl- $\left.\mathrm{CH}_{3}\right)$, $1.93(\mathrm{~m}, 1 \mathrm{H}, 14-\mathrm{Ha}), 1.73\left(\mathrm{~m}, 1 \mathrm{H}, 4^{\prime}-\mathrm{Ha}\right), 1.65\left(\mathrm{~m}, 2 \mathrm{H}, \mathrm{NCH}_{2} \mathrm{CH}_{2} \mathrm{CH}_{2} \mathrm{OH}\right)$, $1.63(\mathrm{~m}, 1 \mathrm{H}, 14-\mathrm{Hb}), 1.52\left(\mathrm{~s}, 3 \mathrm{H}, 6-\mathrm{CH}_{3}\right), 1.38(\mathrm{~m}, 1 \mathrm{H}, 7-\mathrm{Ha}), 1.32(\mathrm{~m}, 1 \mathrm{H}$, $7-\mathrm{Hb}), \quad 1.26\left(\mathrm{~s}, \quad 3 \mathrm{H}, \quad 12-\mathrm{CH}_{3}\right), \quad 1.24\left(\mathrm{~m}, \quad 1 \mathrm{H}, \quad 4^{\prime}-\mathrm{Hb}\right), \quad 1.17(\mathrm{~d}, \quad J=7.0 \mathrm{~Hz}$, $\left.3 \mathrm{H}, 8-\mathrm{CH}_{3}\right), 1.13\left(\mathrm{~d}, J=7.8 \mathrm{~Hz}, 3 \mathrm{H}, 2-\mathrm{CH}_{3}\right), 1.12\left(\mathrm{~d}, J=6.9 \mathrm{~Hz}, 3 \mathrm{H}, 10-\mathrm{CH}_{3}\right)$, $1.10\left(\mathrm{~d}, J=7.4 \mathrm{~Hz}, 3 \mathrm{H}, 4-\mathrm{CH}_{3}\right), 0.78(\mathrm{t}, J=7.2 \mathrm{~Hz}, 3 \mathrm{H}, 15-H)$. ESI-MS $(m / z)$ : $928(\mathrm{M}+\mathrm{H})^{+}$.

2'-O-Benzoyl-11-deoxy-3-des(hexopyranosyloxy)-6-O-methyl-3oxo-11-[3-[(4-trifluoromethylphenylcarbamoyl)oxy]propylamino] erythromycin A 11- $\mathrm{N}, 12-\mathrm{O}$-cyclic carbamate $(5 \mathrm{~h})$

White solid, yield $92 \% .{ }^{1} \mathrm{H}$ NMR $\left(\mathrm{CDCl}_{3}, 400 \mathrm{MHz}\right) \delta 8.08(\mathrm{~d}, J=7.8 \mathrm{~Hz}$, $2 \mathrm{H}$, phenyl- $H 2$, phenyl- $H 6), 7.85(\mathrm{~d}, J=7.2 \mathrm{~Hz}, 2 \mathrm{H}$, phenyl- $H 3$, phenyl- $H 5)$, $7.68(\mathrm{t}, J=6.9 \mathrm{~Hz}, \quad 1 \mathrm{H}$, phenyl- $H 4), \quad 7.49(\mathrm{t}, J=6.0 \mathrm{~Hz}, 2 \mathrm{H}$, phenyl- $H 3$, phenyl- $H 5), 7.44(\mathrm{~d}, J=7.2 \mathrm{~Hz}, 2 \mathrm{H}$, phenyl- $H 2$, phenyl- $H 6), 4.92(\mathrm{dd}, J=2.1$, $8.7 \mathrm{~Hz}, 1 \mathrm{H}, 13-H), 4.57\left(\mathrm{dd}, J=8.0,10.1 \mathrm{~Hz}, 1 \mathrm{H}, 2^{\prime}-H\right), 4.12(\mathrm{~d}, 1 \mathrm{H}, J=10.8 \mathrm{~Hz}$, $5-\mathrm{H}), 3.83(\mathrm{q}, J=7.8 \mathrm{~Hz}, 1 \mathrm{H}, 2-\mathrm{H}), 3.72\left(\mathrm{~m}, 2 \mathrm{H}, \mathrm{CH}_{2} \mathrm{CH}_{2} \mathrm{CH}_{2} \mathrm{OH}\right), 3.60(\mathrm{~s}, 3 \mathrm{H}$, phenyl- $\left.\mathrm{OCH}_{3}\right), 3.55(\mathrm{~d}, J=2.5 \mathrm{~Hz}, 1 \mathrm{H}, 11-H), 3.50\left(\mathrm{~m}, 1 \mathrm{H}, 5^{\prime}-H\right), 3.41(\mathrm{~m}, 1 \mathrm{H}$, $4-H), 3.10(\mathrm{q}, J=7.0 \mathrm{~Hz}, 1 \mathrm{H}, 8-H), 3.05(\mathrm{ql}, J=6.9 \mathrm{~Hz}, 1 \mathrm{H}, 10-H), 2.86(\mathrm{~m}, 2 \mathrm{H}$, $\left.\mathrm{NCH}_{2} \mathrm{CH}_{2} \mathrm{CH}_{2} \mathrm{OH}\right), 2.78\left(\mathrm{~s}, 3 \mathrm{H}, 6-\mathrm{O}-\mathrm{CH}_{3}\right), 2.7\left(\mathrm{~m}, 1 \mathrm{H}, 3^{\prime}-\mathrm{H}\right), 2.27(\mathrm{~s}, 6 \mathrm{H}$, $\left.\mathrm{N}\left(\mathrm{CH}_{3}\right)_{2}\right), 2.24\left(\mathrm{~s}, 3 \mathrm{H}\right.$, phenyl- $\left.\mathrm{CH}_{3}\right), 1.93(\mathrm{~m}, 1 \mathrm{H}, 14-\mathrm{Ha}), 1.73(\mathrm{~m}, 1 \mathrm{H}$, $\left.4^{\prime}-\mathrm{Ha}\right), 1.65\left(\mathrm{~m}, 2 \mathrm{H}, \mathrm{NCH}_{2} \mathrm{CH}_{2} \mathrm{CH}_{2} \mathrm{OH}\right), 1.63(\mathrm{~m}, 1 \mathrm{H}, 14-\mathrm{Hb}), 1.52(\mathrm{~s}, 3 \mathrm{H}$, $\left.6-\mathrm{CH}_{3}\right), 1.38(\mathrm{~m}, 1 \mathrm{H}, 7-\mathrm{Ha}), 1.32(\mathrm{~m}, 1 \mathrm{H}, 7-\mathrm{Hb}), 1.26\left(\mathrm{~s}, 3 \mathrm{H}, 12-\mathrm{CH}_{3}\right)$, $1.24\left(\mathrm{~m}, 1 \mathrm{H}, 4^{\prime}-\mathrm{Hb}\right), 1.17\left(\mathrm{~d}, J=7.0 \mathrm{~Hz}, 3 \mathrm{H}, 8-\mathrm{CH}_{3}\right), 1.13(\mathrm{~d}, J=7.8 \mathrm{~Hz}, 3 \mathrm{H}$, $\left.2-\mathrm{CH}_{3}\right), 1.12\left(\mathrm{~d}, J=6.9 \mathrm{~Hz}, 3 \mathrm{H}, 10-\mathrm{CH}_{3}\right), 1.10\left(\mathrm{~d}, J=7.4 \mathrm{~Hz}, 3 \mathrm{H}, 4-\mathrm{CH}_{3}\right)$, $0.79(\mathrm{t}, J=7.2 \mathrm{~Hz}, 3 \mathrm{H}, 15-\mathrm{H})$. ESI-MS $(\mathrm{m} / \mathrm{z}): 962(\mathrm{M}+\mathrm{H})^{+}$.

General methods for 11-[3-[(arylcarbamoyl)oxy]propylamino]-11deoxy-3-des(hexopyranosyloxy)-6-O-methyl-3-oxoerythromycin A 11-N,12-O-cyclic carbamates (6a-h)

11-[3-[(Arylcarbamoyl)oxy]propylamino]-2'-O-benzoyl-11-deoxy-3-des (hexopyranosyloxy)-6-O-methyl-3-oxoerythromycin A 11- $\mathrm{N}, 12-\mathrm{O}$-cyclic carbamate $(5)(0.5 \mathrm{mmol})$ was dissolved in methanol $(20 \mathrm{ml})$ and the solution was refluxed for $20 \mathrm{~h}$ under nitrogen. The reaction mixture was filtered and concentrated in vacuum. The residue was purified by flash chromatography (cyclohexane-ethyl acetate, 5:2) to afford product $\mathbf{6}$ as a white solid.

11-Deoxy-3-des(hexopyranosyloxy)-6-O-methyl-3-oxo-11-[3[(phenylcarbamoyl)oxy] propylamino] erythromycin A 11- N,12-Ocyclic carbamate $(6 a)$

White solid, yield $95 \% .{ }^{1} \mathrm{H}$ NMR $\left(\mathrm{CDCl}_{3}, 400 \mathrm{MHz}\right) \delta 8.01$ (s, $1 \mathrm{H}$, OCONHphenyl), 7.39(d, $J=7.8 \mathrm{~Hz}, 2 \mathrm{H}$, phenyl- $H 2$, phenyl-H6), $7.29(\mathrm{~d}, J=8.1 \mathrm{~Hz}, 2 \mathrm{H}$, phenyl- $H 3$, phenyl- $H 5), 7.03(\mathrm{t}, J=7.8 \mathrm{~Hz}, 1 \mathrm{H}$, phenyl- $H 4), 4.97(\mathrm{dd}, J=2.1$, $8.7 \mathrm{~Hz}, 1 \mathrm{H}, 13-H), 4.27\left(\mathrm{~d}, J=7.5 \mathrm{~Hz}, 1 \mathrm{H}, \mathrm{I}^{\prime}-H\right), 4.23(\mathrm{~d}, 1 \mathrm{H}, J=8.0 \mathrm{~Hz}, 5-H)$, $3.83(\mathrm{q}, \quad J=7.8 \mathrm{~Hz}, \quad 1 \mathrm{H}, \quad 2-\mathrm{H}), \quad 3.70\left(\mathrm{~m}, \quad 2 \mathrm{H}, \quad \mathrm{CH}_{2} \mathrm{CH}_{2} \mathrm{CH}_{2} \mathrm{OCON}\right), \quad 3.59$ $(\mathrm{d}, J=2.5 \mathrm{~Hz}, 1 \mathrm{H}, 11-\mathrm{H}), 3.50\left(\mathrm{~m}, 1 \mathrm{H}, 5^{\prime}-\mathrm{H}\right), 3.10(\mathrm{~m}, 2 \mathrm{H}, 10-\mathrm{H}, 4-\mathrm{H}), 3.20$ (dd, $\left.J=7.5,8.8 \mathrm{~Hz}, 1 \mathrm{H}, 2^{\prime}-H\right), 3.10(\mathrm{q}, J=7.0 \mathrm{~Hz}, 1 \mathrm{H}, 8-H), 2.86(\mathrm{~m}, 2 \mathrm{H}$, $\left.\mathrm{NCH}_{2} \mathrm{CH}_{2} \mathrm{CH}_{2} \mathrm{OH}\right), 2.61\left(\mathrm{~s}, 3 \mathrm{H}, 6-\mathrm{O}-\mathrm{CH}_{3}\right), 2.48\left(\mathrm{~m}, 1 \mathrm{H}, 3^{\prime}-\mathrm{H}\right), 2.29(\mathrm{~s}, 6 \mathrm{H}, \mathrm{N}$ $\left.\left(\mathrm{CH}_{3}\right)_{2}\right), \quad 1.93(\mathrm{~m}, \quad 1 \mathrm{H}, \quad 14-\mathrm{Ha}), 1.73\left(\mathrm{~m}, 1 \mathrm{H}, \quad 4^{\prime}-\mathrm{Ha}\right), 1.65(\mathrm{~m}, 2 \mathrm{H}$, $\left.\mathrm{NCH}_{2} \mathrm{CH}_{2} \mathrm{CH}_{2} \mathrm{OH}\right), 1.63(\mathrm{~m}, 1 \mathrm{H}, 14-\mathrm{Hb}), 1.47\left(\mathrm{~s}, 3 \mathrm{H}, 12-\mathrm{CH}_{3}\right), 1.38(\mathrm{~m}, 1 \mathrm{H}$, $7-\mathrm{Ha}), 1.34\left(\mathrm{~s}, 3 \mathrm{H}, 6-\mathrm{CH}_{3}\right), 1.32(\mathrm{~m}, 1 \mathrm{H}, 7-\mathrm{Hb}), 1.24\left(\mathrm{~m}, 1 \mathrm{H}, 4^{\prime}-\mathrm{Hb}\right), 1.17$ $\left(\mathrm{d}, J=7.0 \mathrm{~Hz}, 3 \mathrm{H}, 8-\mathrm{CH}_{3}\right), 1.13\left(\mathrm{~d}, J=7.8 \mathrm{~Hz}, 3 \mathrm{H}, 2-\mathrm{CH}_{3}\right), 1.12(\mathrm{~d}, J=6.9 \mathrm{~Hz}$, $\left.3 \mathrm{H}, 10-\mathrm{CH}_{3}\right), 1.10\left(\mathrm{~d}, J=7.4 \mathrm{~Hz}, 3 \mathrm{H}, 4-\mathrm{CH}_{3}\right), 0.85(\mathrm{t}, J=7.2 \mathrm{~Hz}, 3 \mathrm{H}, 15-\mathrm{H})$. $\operatorname{ESI-MS}(m / z): 790(\mathrm{M}+\mathrm{H})^{+}$.

11-Deoxy-3-des(hexopyranosyloxy)-6-O-methyl-11-[3-[(4methylphenylcarbamoyl)oxy]propylamino]-3-oxoerythromycin A 11- $\mathrm{N}, 12-\mathrm{O}$-cyclic carbamate (6b)

White solid, yield $96 \% .{ }^{1} \mathrm{H} \quad \mathrm{NMR}\left(\mathrm{CDCl}_{3}, 400 \mathrm{MHz}\right) \quad \delta \quad 8.9(\mathrm{~s}, \quad 1 \mathrm{H}$, OCONHphenyl), $7.37(\mathrm{~d}, \mathrm{~J}=8.0 \mathrm{~Hz}, 2 \mathrm{H}$, phenyl- $\mathrm{H} 2$, phenyl-H6), 7.06 (d, $J=8.0 \mathrm{~Hz}, 2 \mathrm{H}$, phenyl- $H 3$, phenyl- $H 5), 4.97(\mathrm{dd}, J=2.1,8.7 \mathrm{~Hz}, 1 \mathrm{H}$, $13-H), \quad 4.27\left(\mathrm{~d}, J=7.5 \mathrm{~Hz}, 1 \mathrm{H}, 1^{\prime}-H\right), 4.23(\mathrm{~d}, J=8.0 \mathrm{~Hz}, 1 \mathrm{H}, 5-H), 3.83$ $(\mathrm{q}, \quad J=7.0 \mathrm{~Hz}, \quad 1 \mathrm{H}, \quad 2-\mathrm{H}), \quad 3.70\left(\mathrm{~m}, \quad 2 \mathrm{H}, \quad \mathrm{CH}_{2} \mathrm{CH}_{2} \mathrm{CH}_{2} \mathrm{OCON}\right), \quad 3.59$ $(\mathrm{d}, J=2.5 \mathrm{~Hz}, 1 \mathrm{H}, 11-\mathrm{H}), 3.50\left(\mathrm{~m}, 1 \mathrm{H}, 5^{\prime}-\mathrm{H}\right), 3.10(\mathrm{~m}, 2 \mathrm{H}, 10-\mathrm{H}, 4-\mathrm{H}), 3.20$ (dd, $\left.J=7.5,8.8 \mathrm{~Hz}, 1 \mathrm{H}, 2^{\prime}-H\right), 3.10(\mathrm{q}, J=7.0 \mathrm{~Hz}, 1 \mathrm{H}, 8-\mathrm{H}), 2.86(\mathrm{~m}, 2 \mathrm{H}$, $\left.\mathrm{NCH}_{2} \mathrm{CH}_{2} \mathrm{CH}_{2} \mathrm{OH}\right), 2.61\left(\mathrm{~s}, 3 \mathrm{H}, 6-\mathrm{O}-\mathrm{CH}_{3}\right), 2.48\left(\mathrm{~m}, 1 \mathrm{H}, 3{ }^{\prime}-\mathrm{H}\right), 2.29(\mathrm{~s}, 6 \mathrm{H}$, $\left.\mathrm{N}\left(\mathrm{CH}_{3}\right)_{2}\right), 2.24\left(\mathrm{~s}, 3 \mathrm{H}\right.$, phenyl- $\left.\mathrm{CH}_{3}\right), 1.93(\mathrm{~m}, 1 \mathrm{H}, 14-\mathrm{Ha}), 1.73\left(\mathrm{~m}, 1 \mathrm{H}, 4^{\prime}-\mathrm{Ha}\right)$, $1.65\left(\mathrm{~m}, 2 \mathrm{H}, \mathrm{NCH}_{2} \mathrm{CH}_{2} \mathrm{CH}_{2} \mathrm{O}\right), 1.63(\mathrm{~m}, 1 \mathrm{H}, 14-\mathrm{Hb}), 1.47\left(\mathrm{~s}, 3 \mathrm{H}, 12-\mathrm{CH}_{3}\right), 1.38$ $(\mathrm{m}, 1 \mathrm{H}, 7-\mathrm{Ha}), 1.34\left(\mathrm{~s}, 3 \mathrm{H}, 6-\mathrm{CH}_{3}\right), 1.32(\mathrm{~m}, 1 \mathrm{H}, 7-\mathrm{Hb}), 1.24\left(\mathrm{~m}, 1 \mathrm{H}, 4^{\prime}-\mathrm{Hb}\right)$, $1.17\left(\mathrm{~d}, J=7.0 \mathrm{~Hz}, 3 \mathrm{H}, \quad 8-\mathrm{CH}_{3}\right), \quad 1.13\left(\mathrm{~d}, J=7.8 \mathrm{~Hz}, 3 \mathrm{H}, 2-\mathrm{CH}_{3}\right), \quad 1.12$ $\left(\mathrm{d}, \quad J=6.9 \mathrm{~Hz}, \quad 3 \mathrm{H}, \quad 10-\mathrm{CH}_{3}\right), \quad 1.10\left(\mathrm{~d}, \quad J=7.4 \mathrm{~Hz}, \quad 3 \mathrm{H}, \quad 4-\mathrm{CH}_{3}\right), \quad 0.85$ $(\mathrm{t}, J=7.2 \mathrm{~Hz}, 3 \mathrm{H}, 15-\mathrm{H})$. ESI-MS $(\mathrm{m} / \mathrm{z}): 804(\mathrm{M}+\mathrm{H})^{+}$.

11-Deoxy-3-des(hexopyranosyloxy)-6-O-methyl-11-[3-[(4methoxyphenylcarbamoyl)oxy]propylamino]-3-oxoerythromycin A 11- $N, 12-O$-cyclic carbamate $(6 \mathrm{c})$

White solid, yield $93 \% .{ }^{1} \mathrm{H}$ NMR $\left(\mathrm{CDCl}_{3}, 400 \mathrm{MHz}\right) \quad \delta \quad 8.78(\mathrm{~s}, \quad 1 \mathrm{H}$, OCONHphenyl), $7.47(\mathrm{~d}, J=8.0 \mathrm{~Hz}, 2 \mathrm{H}$, phenyl-H2, phenyl-H6), 6.89 (d, $J=8.0 \mathrm{~Hz}, 2 \mathrm{H}$, phenyl- $H 3$, phenyl-H5), $4.97(\mathrm{dd}, J=2.1,8.7 \mathrm{~Hz}, 1 \mathrm{H}$, $13-H), 4.27\left(\mathrm{~d}, J=7.5 \mathrm{~Hz}, 1 \mathrm{H}, 1^{\prime}-H\right), 4.23(\mathrm{~d}, J=8.0 \mathrm{~Hz}, 1 \mathrm{H}, 5-H), 3.83$ (q, $J=7.8 \mathrm{~Hz}, 1 \mathrm{H}, 2-\mathrm{H}), 3.70\left(\mathrm{~m}, 2 \mathrm{H}, \mathrm{CH}_{2} \mathrm{CH}_{2} \mathrm{CH}_{2} \mathrm{OCON}\right), 3.60(\mathrm{~s}, 3 \mathrm{H}$, phenyl- $\left.-\mathrm{OCH}_{3}\right), \quad 3.59(\mathrm{~d}, J=2.5 \mathrm{~Hz}, 1 \mathrm{H}, 11-\mathrm{H}), 3.50\left(\mathrm{~m}, 1 \mathrm{H}, 5^{\prime}-\mathrm{H}\right), 3.10$ $(\mathrm{m}, 2 \mathrm{H}, 10-\mathrm{H}, 4-H), 3.20\left(\mathrm{dd}, J=7.5,8.8 \mathrm{~Hz}, 1 \mathrm{H}, 2^{\prime}-H\right), 3.10(\mathrm{q}, J=7.0 \mathrm{~Hz}$, $1 \mathrm{H}, 8-\mathrm{H}), 2.86\left(\mathrm{~m}, 2 \mathrm{H}, \mathrm{NCH}_{2} \mathrm{CH}_{2} \mathrm{CH}_{2} \mathrm{OH}\right), 2.61\left(\mathrm{~s}, 3 \mathrm{H}, 6-\mathrm{O}-\mathrm{CH}_{3}\right), 2.48(\mathrm{~m}, 1 \mathrm{H}$, $\left.3^{\prime}-\mathrm{H}\right), 2.29\left(\mathrm{~s}, 6 \mathrm{H}, \mathrm{N}\left(\mathrm{CH}_{3}\right)_{2}\right), 1.93(\mathrm{~m}, 1 \mathrm{H}, 14-\mathrm{Ha}), 1.73\left(\mathrm{~m}, 1 \mathrm{H}, 4^{\prime}-\mathrm{Ha}\right), 1.65$ $\left(\mathrm{m}, 2 \mathrm{H}, \mathrm{NCH}_{2} \mathrm{CH}_{2} \mathrm{CH}_{2} \mathrm{O}\right), 1.63(\mathrm{~m}, 1 \mathrm{H}, 14-\mathrm{Hb}), 1.47\left(\mathrm{~s}, 3 \mathrm{H}, 12-\mathrm{CH}_{3}\right), 1.38$ $(\mathrm{m}, 1 \mathrm{H}, 7-\mathrm{Ha}), 1.34\left(\mathrm{~s}, 3 \mathrm{H}, 6-\mathrm{CH}_{3}\right), 1.32(\mathrm{~m}, 1 \mathrm{H}, 7-\mathrm{Hb}), 1.24\left(\mathrm{~m}, 1 \mathrm{H}, 4^{\prime}-\mathrm{Hb}\right)$, $1.17\left(\mathrm{~d}, J=7.0 \mathrm{~Hz}, \quad 3 \mathrm{H}, \quad 8-\mathrm{CH}_{3}\right), \quad 1.13\left(\mathrm{~d}, J=7.8 \mathrm{~Hz}, 3 \mathrm{H}, \quad 2-\mathrm{CH}_{3}\right), \quad 1.12$ $\left(\mathrm{d}, \quad J=6.9 \mathrm{~Hz}, \quad 3 \mathrm{H}, \quad 10-\mathrm{CH}_{3}\right), \quad 1.10\left(\mathrm{~d}, \quad J=7.4 \mathrm{~Hz}, \quad 3 \mathrm{H}, \quad 4-\mathrm{CH}_{3}\right), \quad 0.84$ $(\mathrm{t}, J=7.2 \mathrm{~Hz}, 3 \mathrm{H}, 15-\mathrm{H})$. ESI-MS $(\mathrm{m} / \mathrm{z}): 820(\mathrm{M}+\mathrm{H})^{+}$.

11-Deoxy-3-des(hexopyranosyloxy)-6-O-methyl-11-[3-[(4-nitrophenylcarbamoyl)oxy]propylamino]-3-oxoerythromycin A 11-N, 12-O-cyclic carbamate $(6 \mathrm{~d})$

White solid, yield $86 \% .{ }^{1} \mathrm{H}$ NMR $\left(\mathrm{CDCl}_{3}, 400 \mathrm{MHz}\right) \delta 9.08(\mathrm{~s}, 1 \mathrm{H}$, OCONHphenyl), $8.05(\mathrm{~d}, J=8.2 \mathrm{~Hz}, 2 \mathrm{H}$, phenyl- $\mathrm{H} 2$, phenyl-H6), 7.63 (d, $J=8.2 \mathrm{~Hz}, 2 \mathrm{H}$, phenyl- $H 3$, phenyl- $H 5), 4.97(\mathrm{dd}, J=2.1,8.7 \mathrm{~Hz}, 1 \mathrm{H}$, $13-H), \quad 4.27\left(\mathrm{~d}, J=7.5 \mathrm{~Hz}, 1 \mathrm{H}, 1^{\prime}-H\right), 4.23(\mathrm{~d}, J=8.0 \mathrm{~Hz}, 1 \mathrm{H}, 5-H), 3.83$ $(\mathrm{q}, \quad J=7.8 \mathrm{~Hz}, \quad 1 \mathrm{H}, \quad 2-\mathrm{H}), \quad 3.70\left(\mathrm{~m}, \quad 2 \mathrm{H}, \quad \mathrm{CH}_{2} \mathrm{CH}_{2} \mathrm{CH}_{2} \mathrm{OCON}\right), \quad 3.59$ $(\mathrm{d}, J=2.5 \mathrm{~Hz}, 1 \mathrm{H}, 11-\mathrm{H}), 3.50\left(\mathrm{~m}, 1 \mathrm{H}, 5^{\prime}-\mathrm{H}\right), 3.10(\mathrm{~m}, 2 \mathrm{H}, 10-\mathrm{H}, 4-\mathrm{H}), 3.20$ (dd, $\left.J=7.5,8.8 \mathrm{~Hz}, 1 \mathrm{H}, 2^{\prime}-H\right), 3.10(\mathrm{q}, J=7.0 \mathrm{~Hz}, 1 \mathrm{H}, 8-H), 2.86(\mathrm{~m}, 2 \mathrm{H}$, $\left.\mathrm{NCH}_{2} \mathrm{CH}_{2} \mathrm{CH}_{2} \mathrm{O}\right), 2.61\left(\mathrm{~s}, 3 \mathrm{H}, 6-\mathrm{O}-\mathrm{CH}_{3}\right), 2.48\left(\mathrm{~m}, 1 \mathrm{H}, 3^{\prime}-\mathrm{H}\right), 2.29(\mathrm{~s}, 6 \mathrm{H}, \mathrm{N}$ $\left.\left(\mathrm{CH}_{3}\right)_{2}\right), \quad 1.93(\mathrm{~m}, 1 \mathrm{H}, 14-\mathrm{Ha}), 1.73\left(\mathrm{~m}, 1 \mathrm{H}, \quad 4^{\prime}-\mathrm{Ha}\right), \quad 1.65(\mathrm{~m}, 2 \mathrm{H}$, $\left.\mathrm{NCH}_{2} \mathrm{CH}_{2} \mathrm{CH}_{2} \mathrm{O}\right), 1.63(\mathrm{~m}, 1 \mathrm{H}, 14-\mathrm{Hb}), 1.47\left(\mathrm{~s}, 3 \mathrm{H}, 12-\mathrm{CH}_{3}\right), 1.38(\mathrm{~m}, 1 \mathrm{H}$, 7- $\mathrm{Ha}), 1.34\left(\mathrm{~s}, 3 \mathrm{H}, 6-\mathrm{CH}_{3}\right), 1.32(\mathrm{~m}, 1 \mathrm{H}, 7-\mathrm{Hb}), 1.24\left(\mathrm{~m}, 1 \mathrm{H}, 4^{\prime}-\mathrm{Hb}\right), 1.17$ $\left(\mathrm{d}, J=7.0 \mathrm{~Hz}, 3 \mathrm{H}, 8-\mathrm{CH}_{3}\right), 1.13\left(\mathrm{~d}, J=7.8 \mathrm{~Hz}, 3 \mathrm{H}, 2-\mathrm{CH}_{3}\right), 1.12(\mathrm{~d}, J=6.9 \mathrm{~Hz}$, $\left.3 \mathrm{H}, 10-\mathrm{CH}_{3}\right), 1.10\left(\mathrm{~d}, J=7.4 \mathrm{~Hz}, 3 \mathrm{H}, 4-\mathrm{CH}_{3}\right), 0.84(\mathrm{t}, J=7.2 \mathrm{~Hz}, 3 \mathrm{H}, 15-\mathrm{H})$. $\operatorname{ESI-MS}(m / z): 835(\mathrm{M}+\mathrm{H})^{+}$.

11-Deoxy-3-des(hexopyranosyloxy)-6-O-methyl-11-[3-[(3-methylphenylcarbamoyl)oxy]propylamino]-3-oxoerythromycin A 11- $N$, 12-O-cyclic carbamate $(6 \mathrm{e})$

White solid, yield 96\%. ${ }^{1} \mathrm{H}$ NMR $\left(\mathrm{CDCl}_{3}, 400 \mathrm{MHz}\right) \quad \delta \quad 9.61(\mathrm{~s}, \quad 1 \mathrm{H}$, OCONHphenyl), 7.50(s, $1 \mathrm{H}$, phenyl-H2), $7.45(\mathrm{~d}, J=7.7 \mathrm{~Hz}, 1 \mathrm{H}$, phenyl-H6), $7.04(\mathrm{~d}, J=7.7 \mathrm{~Hz}, 1 \mathrm{H}$, phenyl-H5), $6.82(\mathrm{~d}, J=7.7 \mathrm{~Hz}, 1 \mathrm{H}$, phenyl- $H 4)$, $4.97(\mathrm{dd}, J=2.1,8.7 \mathrm{~Hz}, 1 \mathrm{H}, 13-H), 4.27\left(\mathrm{~d}, J=7.5 \mathrm{~Hz}, 1 \mathrm{H}, 1^{\prime}-H\right), 4.23$ $(\mathrm{d}, \quad 1 \mathrm{H}, J=8.0 \mathrm{~Hz}, 5-\mathrm{H}), \quad 3.83(\mathrm{q}, J=7.8 \mathrm{~Hz}, \quad 1 \mathrm{H}, 2-\mathrm{H}), 3.70(\mathrm{~m}, 2 \mathrm{H}$, 
$\left.\mathrm{CH}_{2} \mathrm{CH}_{2} \mathrm{CH}_{2} \mathrm{OCON}\right), 3.59(\mathrm{~d}, J=2.5 \mathrm{~Hz}, 1 \mathrm{H}, 11-\mathrm{H}), 3.50\left(\mathrm{~m}, 1 \mathrm{H}, 5^{\prime}-\mathrm{H}\right), 3.10$ $(\mathrm{m}, 2 \mathrm{H}, 10-\mathrm{H}, 4-H), 3.20\left(\mathrm{dd}, J=7.5,8.8 \mathrm{~Hz}, 1 \mathrm{H}, 2^{\prime}-H\right), 3.10(\mathrm{q}, J=7.0 \mathrm{~Hz}$, $1 \mathrm{H}, 8-\mathrm{H}), 2.86\left(\mathrm{~m}, 2 \mathrm{H}, \mathrm{NCH}_{2} \mathrm{CH}_{2} \mathrm{CH}_{2} \mathrm{OH}\right), 2.61\left(\mathrm{~s}, 3 \mathrm{H}, 6-\mathrm{O}-\mathrm{CH}_{3}\right), 2.48(\mathrm{~m}, 1 \mathrm{H}$, $\left.3^{\prime}-\mathrm{H}\right), 2.29\left(\mathrm{~s}, 6 \mathrm{H}, \mathrm{N}\left(\mathrm{CH}_{3}\right) 2\right), 2.24\left(\mathrm{~s}, 3 \mathrm{H}\right.$, phenyl- $\left.\mathrm{CH}_{3}\right), 1.93(\mathrm{~m}, 1 \mathrm{H}, 14-\mathrm{Ha})$, $1.73\left(\mathrm{~m}, 1 \mathrm{H}, 4^{\prime}-\mathrm{Ha}\right), 1.65\left(\mathrm{~m}, 2 \mathrm{H}, \mathrm{NCH}_{2} \mathrm{CH}_{2} \mathrm{CH}_{2} \mathrm{O}\right), 1.63(\mathrm{~m}, 1 \mathrm{H}, 14-\mathrm{Hb}), 1.47$ $\left(\mathrm{s}, 3 \mathrm{H}, 12-\mathrm{CH}_{3}\right), 1.38(\mathrm{~m}, 1 \mathrm{H}, 7-\mathrm{Ha}), 1.34\left(\mathrm{~s}, 3 \mathrm{H}, 6-\mathrm{CH}_{3}\right), 1.32(\mathrm{~m}, 1 \mathrm{H}, 7-\mathrm{Hb})$, $1.24\left(\mathrm{~m}, 1 \mathrm{H}, 4^{\prime}-\mathrm{Hb}\right), 1.17\left(\mathrm{~d}, J=7.0 \mathrm{~Hz}, 3 \mathrm{H}, 8-\mathrm{CH}_{3}\right), 1.13(\mathrm{~d}, J=7.8 \mathrm{~Hz}, 3 \mathrm{H}$, $\left.2-\mathrm{CH}_{3}\right), 1.12\left(\mathrm{~d}, J=6.9 \mathrm{~Hz}, 3 \mathrm{H}, 10-\mathrm{CH}_{3}\right), 1.10\left(\mathrm{~d}, J=7.4 \mathrm{~Hz}, 3 \mathrm{H}, 4-\mathrm{CH}_{3}\right)$, $0.84(\mathrm{t}, J=7.2 \mathrm{~Hz}, 3 \mathrm{H}, 15-\mathrm{H})$. ESI-MS $(\mathrm{m} / \mathrm{z}): 804(\mathrm{M}+\mathrm{H})$

\section{1-[3-[(2-Chlorophenylcarbamoyl)oxy]propylamino]-11-deoxy-3- des(hexopyranosyloxy)-6-O-methyl-3-oxoerythromycin A 11- $\mathrm{N}$, 12-O-cyclic carbamate (6f)}

White solid, yield $91 \% .{ }^{1} \mathrm{H}$ NMR $\left(\mathrm{CDCl}_{3}, 400 \mathrm{MHz}\right) \delta 8.10(\mathrm{t}, J=6.9 \mathrm{~Hz}, 1 \mathrm{H}$, phenyl-H6), 7.85(s, 1H, phenyl-H2), 7.78(s, 1H, OCONHphenyl), 7.15 $(\mathrm{t}, \quad J=7.7 \mathrm{~Hz}, \quad 1 \mathrm{H}, \quad$ phenyl- $H 3), \quad 7.00(\mathrm{t}, J=8.0 \mathrm{~Hz}, \quad 1 \mathrm{H}, \quad$ phenyl- $H 5)$, $6.92(\mathrm{t}, J=8.0 \mathrm{~Hz}, 1 \mathrm{H}$, phenyl-H4), 4.97(dd, $J=2.1,8.7 \mathrm{~Hz}, 1 \mathrm{H}, 13-H), 4.27$ $\left(\mathrm{d}, J=7.5 \mathrm{~Hz}, 1 \mathrm{H}, 1^{\prime}-H\right), 4.23(\mathrm{~d}, J=8.0 \mathrm{~Hz}, 1 \mathrm{H}, 5-H), 3.83(\mathrm{q}, J=7.8 \mathrm{~Hz}, 1 \mathrm{H}$, 2- $\mathrm{H}), 3.70\left(\mathrm{~m}, 2 \mathrm{H}, \mathrm{CH}_{2} \mathrm{CH}_{2} \mathrm{CH}_{2} \mathrm{OCON}\right), 3.59(\mathrm{~d}, J=2.5 \mathrm{~Hz}, 1 \mathrm{H}, 11-\mathrm{H}), 3.50$ $\left(\mathrm{m}, 1 \mathrm{H}, 5^{\prime}-H\right), 3.10(\mathrm{~m}, 2 \mathrm{H}, 10-H, 4-H), 3.20\left(\mathrm{dd}, J=7.5,8.8 \mathrm{~Hz}, 1 \mathrm{H}, 2^{\prime}-H\right)$ $3.10(\mathrm{q}, J=7.0 \mathrm{~Hz}, 1 \mathrm{H}, 8-\mathrm{H}), 2.86\left(\mathrm{~m}, 2 \mathrm{H}, \mathrm{NCH}_{2} \mathrm{CH}_{2} \mathrm{CH}_{2} \mathrm{O}\right), 2.61(\mathrm{~s}, 3 \mathrm{H}$, 6-O- $\left.\mathrm{CH}_{3}\right), 2.48\left(\mathrm{~m}, 1 \mathrm{H}, 3^{\prime}-\mathrm{H}\right), 2.29\left(\mathrm{~s}, 6 \mathrm{H}, \mathrm{N}\left(\mathrm{CH}_{3}\right) 2\right), 1.93(\mathrm{~m}, 1 \mathrm{H}, 14-\mathrm{Ha})$, $1.73\left(\mathrm{~m}, 1 \mathrm{H}, 4^{\prime}-\mathrm{Ha}\right), 1.65\left(\mathrm{~m}, 2 \mathrm{H}, \mathrm{NCH}_{2} \mathrm{CH}_{2} \mathrm{CH}_{2} \mathrm{O}\right), 1.63(\mathrm{~m}, 1 \mathrm{H}, 14-\mathrm{Hb}), 1.47$ $\left(\mathrm{s}, 3 \mathrm{H}, 12-\mathrm{CH}_{3}\right), 1.38(\mathrm{~m}, 1 \mathrm{H}, 7-\mathrm{Ha}), 1.34\left(\mathrm{~s}, 3 \mathrm{H}, 6-\mathrm{CH}_{3}\right), 1.32(\mathrm{~m}, 1 \mathrm{H}, 7-\mathrm{Hb})$, $1.24\left(\mathrm{~m}, 1 \mathrm{H}, 4^{\prime}-\mathrm{Hb}\right), 1.17\left(\mathrm{~d}, J=7.0 \mathrm{~Hz}, 3 \mathrm{H}, 8-\mathrm{CH}_{3}\right), 1.13(\mathrm{~d}, J=7.8 \mathrm{~Hz}, 3 \mathrm{H}$ $\left.2-\mathrm{CH}_{3}\right), 1.12\left(\mathrm{~d}, J=6.9 \mathrm{~Hz}, 3 \mathrm{H}, 10-\mathrm{CH}_{3}\right), 1.10\left(\mathrm{~d}, J=7.4 \mathrm{~Hz}, 3 \mathrm{H}, 4-\mathrm{CH}_{3}\right)$ $0.84(\mathrm{t}, J=7.2 \mathrm{~Hz}, 3 \mathrm{H}, 15-\mathrm{H})$. ESI-MS $(\mathrm{m} / \mathrm{z}): 824(\mathrm{M}+\mathrm{H})^{+}$

\section{1-[3-[(3-Chlorophenylcarbamoyl)oxy]propylamino]-11-deoxy-3- des(hexopyranosyloxy)-6-O-methyl-3-oxoerythromycin A 11- $\mathrm{N}$, 12-O-cyclic carbamate $(6 \mathrm{~g})$}

White solid, yield $90 \% .{ }^{1} \mathrm{H}$ NMR $\left(\mathrm{CDCl}_{3}, 400 \mathrm{MHz}\right) \delta 10.61(\mathrm{~s}, 1 \mathrm{H}$ OCONHphenyl), 7.85(s, $1 \mathrm{H}$, phenyl- $H 2$ ), $7.55(\mathrm{~d}, J=7.7 \mathrm{~Hz}, 1 \mathrm{H}$, phenyl- $H 6$ ), $7.21(\mathrm{~d}, J=7.7 \mathrm{~Hz}, 1 \mathrm{H}$, phenyl- $H 5), 7.03(\mathrm{~d}, J=7.7 \mathrm{~Hz}, 1 \mathrm{H}$, phenyl- $H 4), 4.97$ $(\mathrm{dd}, J=2.1,8.7 \mathrm{~Hz}, 1 \mathrm{H}, 13-H), 4.27\left(\mathrm{~d}, J=7.5 \mathrm{~Hz}, 1 \mathrm{H}, l^{\prime}-H\right), 4.23(\mathrm{~d}, J=8.0 \mathrm{~Hz}$ $1 \mathrm{H}, 5-\mathrm{H}), 3.83(\mathrm{q}, J=7.8 \mathrm{~Hz}, 1 \mathrm{H}, 2-\mathrm{H}), 3.70\left(\mathrm{~m}, 2 \mathrm{H}, \mathrm{CH}_{2} \mathrm{CH}_{2} \mathrm{CH}_{2} \mathrm{OCON}\right)$, $3.59(\mathrm{~d}, J=2.5 \mathrm{~Hz}, 1 \mathrm{H}, 11-\mathrm{H}), 3.50\left(\mathrm{~m}, 1 \mathrm{H}, 5^{\prime}-H\right), 3.10(\mathrm{~m}, 2 \mathrm{H}, 10-\mathrm{H}, 4-H), 3.20$ $\left(\mathrm{dd}, J=7.5,8.8 \mathrm{~Hz}, 1 \mathrm{H}, 2^{\prime}-H\right), 3.10(\mathrm{q}, J=7.0 \mathrm{~Hz}, 1 \mathrm{H}, 8-H), 2.86(\mathrm{~m}, 2 \mathrm{H}$, $\mathrm{NCH}_{2} \mathrm{CH}_{2} \mathrm{CH}_{2} \mathrm{O}$ ), 2.61(s, 3H, 6-O-CH $\left.\mathrm{CH}_{3}\right), 2.48\left(\mathrm{~m}, 1 \mathrm{H}, 3^{\prime}-\mathrm{H}\right), 2.29(\mathrm{~s}, 6 \mathrm{H}, \mathrm{N}$ $\left.\left(\mathrm{CH}_{3}\right) 2\right), \quad 1.93(\mathrm{~m}, \quad 1 \mathrm{H}, 14-\mathrm{Ha}), 1.73\left(\mathrm{~m}, 1 \mathrm{H}, \quad 4^{\prime}-\mathrm{Ha}\right), \quad 1.65(\mathrm{~m}, \quad 2 \mathrm{H}$ $\left.\mathrm{NCH}_{2} \mathrm{CH}_{2} \mathrm{CH}_{2} \mathrm{O}\right), 1.63(\mathrm{~m}, 1 \mathrm{H}, 14-\mathrm{Hb}), 1.47\left(\mathrm{~s}, 3 \mathrm{H}, 12-\mathrm{CH}_{3}\right), 1.38(\mathrm{~m}, 1 \mathrm{H}$ $7-\mathrm{Ha}), \quad 1.34\left(\mathrm{~s}, 3 \mathrm{H}, 6-\mathrm{CH}_{3}\right), 1.32(\mathrm{~m}, 1 \mathrm{H}, 7-\mathrm{Hb}), 1.24\left(\mathrm{~m}, 1 \mathrm{H}, 4^{\prime}-\mathrm{Hb}\right)$, $1.17\left(\mathrm{~d}, \quad J=7.0 \mathrm{~Hz}, \quad 3 \mathrm{H}, \quad 8-\mathrm{CH}_{3}\right), \quad 1.13\left(\mathrm{~d}, J=7.8 \mathrm{~Hz}, 3 \mathrm{H}, \quad 2-\mathrm{CH}_{3}\right), \quad 1.12$ $\left(\mathrm{d}, \quad J=6.9 \mathrm{~Hz}, \quad 3 \mathrm{H}, \quad 10-\mathrm{CH}_{3}\right), \quad 1.10\left(\mathrm{~d}, \quad J=7.4 \mathrm{~Hz}, \quad 3 \mathrm{H}, \quad 4-\mathrm{CH}_{3}\right), \quad 0.85$ $(\mathrm{t}, J=7.2 \mathrm{~Hz}, 3 \mathrm{H}, 15-H)$. ESI-MS $(m / z): 824(\mathrm{M}+\mathrm{H})^{+}$.

11-Deoxy-3-des(hexopyranosyloxy)-6-O-methyl-3-oxo-11-[3-[(4trifluoromethylphenylcarbamoyl)oxy]propylamino] erythromycin A 11-N,12-O-cyclic carbamate $(6 \mathrm{~h})$

White solid, yield $93 \% .{ }^{1} \mathrm{H}$ NMR $\left(\mathrm{CDCl}_{3}, 400 \mathrm{MHz}\right) \delta 7.85(\mathrm{~d}, J=7.2 \mathrm{~Hz}, 2 \mathrm{H}$, phenyl- $H 3$, phenyl- $H 5), 7.68(\mathrm{t}, J=6.9 \mathrm{~Hz}, 1 \mathrm{H}$, phenyl- $H 4), 7.44(\mathrm{~d}, J=7.2 \mathrm{~Hz}$, $2 \mathrm{H}$, phenyl-H2, phenyl-H6), $4.97(\mathrm{dd}, J=2.1,8.7 \mathrm{~Hz}, 1 \mathrm{H}, 13-\mathrm{H}), 4.27$ $\left(\mathrm{d}, J=7.5 \mathrm{~Hz}, 1 \mathrm{H}, 1^{\prime}-H\right), 4.23(\mathrm{~d}, J=8.0 \mathrm{~Hz}, 1 \mathrm{H}, 5-H), 3.83(\mathrm{q}, J=7.8 \mathrm{~Hz}$, $1 \mathrm{H}, 2-\mathrm{H}), 3.70\left(\mathrm{~m}, 2 \mathrm{H}, \mathrm{CH}_{2} \mathrm{CH}_{2} \mathrm{CH}_{2} \mathrm{OCON}\right), 3.59(\mathrm{~d}, J=2.5 \mathrm{~Hz}, 1 \mathrm{H}, 11-\mathrm{H})$ $3.50\left(\mathrm{~m}, 1 \mathrm{H}, 5^{\prime}-H\right), 3.10(\mathrm{~m}, 2 \mathrm{H}, 10-H, 4-H), 3.20\left(\mathrm{dd}, J=7.5,8.8 \mathrm{~Hz}, 1 \mathrm{H}, 2^{\prime}-H\right)$ $3.10(\mathrm{q}, J=7.0 \mathrm{~Hz}, 1 \mathrm{H}, 8-\mathrm{H}), 2.86\left(\mathrm{~m}, 2 \mathrm{H}, \mathrm{NCH}_{2} \mathrm{CH}_{2} \mathrm{CH}_{2} \mathrm{O}\right), 2.61(\mathrm{~s}, 3 \mathrm{H}$, 6-O- $\left.\mathrm{CH}_{3}\right), 2.48\left(\mathrm{~m}, 1 \mathrm{H}, 3^{\prime}-\mathrm{H}\right), 2.29\left(\mathrm{~s}, 6 \mathrm{H}, \mathrm{N}\left(\mathrm{CH}_{3}\right)_{2}\right), 1.93(\mathrm{~m}, 1 \mathrm{H}, 14-\mathrm{Ha})$, $1.73\left(\mathrm{~m}, 1 \mathrm{H}, 4^{\prime}-\mathrm{Ha}\right), 1.65\left(\mathrm{~m}, 2 \mathrm{H}, \mathrm{NCH}_{2} \mathrm{CH}_{2} \mathrm{CH}_{2} \mathrm{O}\right), 1.63(\mathrm{~m}, 1 \mathrm{H}, 14-\mathrm{Hb}), 1.47$ $\left(\mathrm{s}, 3 \mathrm{H}, 12-\mathrm{CH}_{3}\right), 1.38(\mathrm{~m}, 1 \mathrm{H}, 7-\mathrm{Ha}), 1.34\left(\mathrm{~s}, 3 \mathrm{H}, 6-\mathrm{CH}_{3}\right), 1.32(\mathrm{~m}, 1 \mathrm{H}, 7-\mathrm{Hb})$, $1.24\left(\mathrm{~m}, 1 \mathrm{H}, 4^{\prime}-\mathrm{Hb}\right), 1.17\left(\mathrm{~d}, J=7.0 \mathrm{~Hz}, 3 \mathrm{H}, 8-\mathrm{CH}_{3}\right), 1.13(\mathrm{~d}, J=7.8 \mathrm{~Hz}, 3 \mathrm{H}$, $\left.2-\mathrm{CH}_{3}\right), 1.12\left(\mathrm{~d}, J=6.9 \mathrm{~Hz}, 3 \mathrm{H}, 10-\mathrm{CH}_{3}\right), 1.10\left(\mathrm{~d}, J=7.4 \mathrm{~Hz}, 3 \mathrm{H}, 4-\mathrm{CH}_{3}\right)$, $0.85(\mathrm{t}, J=7.2 \mathrm{~Hz}, 3 \mathrm{H}, 15-\mathrm{H})$. ESI-MS $(\mathrm{m} / \mathrm{z}): 858(\mathrm{M}+\mathrm{H})^{+}$.

\section{CONFLICT OF INTEREST}

The authors declare no conflict of interest.

\section{ACKNOWLEDGEMENTS}

This research was supported by Shandong Xinhua Pharmaceutical Co., Ltd., and National Natural Science Foundation of China (30801427).

1 Metz, M. \& Shlaes, D. M. Eight more ways to deal with antibiotic resistance. Antimicrob. Agents Chemother. 58, 4253-4256 (2014).

2 McManus, B. A. et al. Susceptibility of methicillin-resistant Staphylococci clinical isolates to netilmicin and other antibiotics commonly used in ophthalmic therapy. Curr. Eye Res. 38, 811-816 (2013)

3 McManus, B. A. et al. Comparative genotypes, Staphylococcal Cassette Chromosome mec (SCCmec) genes and antimicrobial resistance amongst Staphylococcus epidermidis and Staphylococcus haemolyticus isolates from infections in humans and companion animals. PLOS ONE. 10, e0138079 (2015)

4 Farrell, D. J. et al. Emergence and spread of Streptococcus pneumoniae with erm(B) and mef(A) resistance. Emerg. Infect. Dis. 11, 851-858 (2005)

5 Morimoto, S., Takahashi, Y., Watanabe, Y. \& Omura, S. Chemical modification of erythromycins. I. Synthesis and antibacterial activity of 6-0-methylerythromycins A. J. Antibiot. 37, 187-189 (1984).

6 Zhanel, G. G. et al. Review of macrolides and ketolides focus on respiratory tract infections. Drugs 61, 443-498 (2001).

7 Leclercq, R. \& Courvalin, P. Bacterial resistance to macrolide, lincosamide, and streptogramin antibiotics by target modification. Antimicrob. Agents Chemother. 35 1267-1272 (1991).

8 Leclercq, R. \& Courvalin, P. Resistance to macrolides and related antibiotics in S. pneumoniae. Antimicrob. Agents Chemother. 46, 2727-2734 (2002).

9 Denis, A. et al. Synthesis and antibacterial activity of HMR 3647 a new ketolide highly potent against erythromycin-resistant and susceptible pathogens. Bioorg. Med. Chem. Lett. 9, 3075-3080 (1999).

$10 \mathrm{Or}, \mathrm{Y}$. S et al. Design, synthesis, and antimicrobial activity of 6-0-substituted ketolides active against resistant respiratory tract pathogens. J. Med. Chem. 43 1045-1049 (2000).

11 Champney, W. S. \& Tober, C. L. Structure-activity relationship for six ketolide antibiotics. Curr Microbiol 42, 203-210 (2001).

12 Zhanel, G. G. et al. The ketolides: a critical review. Drugs 62, 1771-1804 (2002).

13 Keyes, R. F. et al. Synthesis and antibacterial activity of 6-0-arylbutynyl ketolides with improved activity against some key erythromycin-resistant pathogens. J. Med. Chem 46, 1795-1798 (2003).

14 Yong, $\mathrm{H}$. et al. Design, synthesis and structure-activity relationships of 6-0-arylpropargyl diazalides with potent activity against multidrug-resistant Streptococcus pneumoniae. Bioorg. Med. Chem. Lett. 15, 2653-2658 (2005).

15 Sugimoto, T. et al. Synthesis and antibacterial activity of 6-0-(heteroaryl-isoxazolyl) propynyl 2-fluoro ketolides. Bioorg. Med. Chem. Lett 22, 5739-5743 (2012).

16 Nomura, T., Yasukata, T., Narukawa, Y. \& Uotani, K. 9-0xime-3-ketolides: modification at the $\mathrm{C}$-11,12-diol moiety and antibacterial activities against key respiratory pathogens. Bioorg. Med. Chem. 13, 6054-6063 (2005).

17 Beebe, X. et al. Synthesis and antibacterial activity of 6-0-arylpropargyl-9-oxime-11, 12-carbamate ketolides. Bioorg. Med. Chem. Lett. 14, 2417-2421 (2004).

18 Liang, J.-H. et al. Synthesis and antibacterial activities of 6-0-methylerythromycin A 9-0-(3-aryl-2-propenyl) oxime ketolide, 2,3-enol ether, and alkylide analogues. Eur. J. Med. Chem. 45, 3627-3635 (2010).

19 Hunziker, D. et al. Novel ketolide antibiotics with a fused five-membered lactone ring-synthesis, physicochemical and antimicrobial properties. Bioorg. Med. Chem. 12, 3503-3519 (2004)

20 Zhu, B. et al. Synthesis and antibacterial activity of 3-keto-6-0-carbamoyl-11,12-cyclic thiocarbamate erythromycin A derivatives. Bioorg. Med. Chem. Lett. 17, 3900-3904 (2007).

21 Elliott, R. L. et al. Anhydrolide Macrolides. 1. Synthesis and Antibacterial Activity of 2,3-Anhydro-6-0-methyl-11,12-carbamate Erythromycin A Analogues. J. Med. Chem. 41, 1651-1659 (1998).

22 Wang, G. et al. 6-11 Bridged oxime erythromycin derivatives WO 2006119313 , accessed on 9 November 2006.

23 Sato, T. et al. In vitro antibacterial activity of modithromycin, a novel 6, 11-bridged bicyclolide, against respiratory pathogens, including macrolide-resistant gram-positive cocci. Antimicrob. Agents Chemother. 55, 1588-1593 (2011)

24 Or, Y. S. et al. 6-11 Bicyclic ketolide derivatives WO 2005061525, accessed on 7 July 2005.

25 Downey, A. M. \& Cairo, C. W. Synthesis of $\alpha$-brominated phosphonates and their application as phosphate bioisosteres. Med. Chem. Commun. 5, 1619-1633 (2014).

26 Agours, C. et al. Synthesis and antibacterial activity of ketolides (6-0-Methyl-3oxoerythromycin derivatives): a new class of antibacterials highly potent against macrolides-resistant and susceptible respiratiory pathogents. J. Med. Chem. 41, 4080-4100 (1998). 